\title{
Unit commitment constraints in long-term planning models: relevance, pitfalls and the role of assumptions on flexibility
}

\author{
Kris Poncelet, Erik Delarue and William D'haeseleer \\ KU Leuven Department of Mechanical Engineering, TME Division \\ EnergyVille.
}

\begin{abstract}
In the context of an increasing penetration of intermittent renewable energy sources, the impact of neglecting unit commitment constraints in generation expansion planning models has been widely assessed and demonstrated in the literature. However, the focus is often on thermal generators being the only source of flexibility, and the sensitivity to certain assumptions (e.g., system requirements and technical characteristics) has not been investigated. This paper contributes to the existing literature by revisiting the relevance of considering unit commitment constraints in generation expansion planning models for varying assumptions regarding both the available flexibility and the need for operating reserve requirements. The results indicate that if storage technologies are considered, integrating technical constraints has only a minor impact on both overall cost projections and most investments, with the exception of investments in storage technologies themselves. Furthermore, the investments in storage technologies are shown to be highly sensitive to the assumptions made regarding the assumed flexibility of thermal generators and the future need for operating reserves. These sensitivities are shown to be of an order of magnitude higher or of an equal order of magnitude compared to the impact of integrating technical constraints with continuous rather than integer commitment variables.
\end{abstract}

Keywords: Generation expansion planning, Long-term planning models, Energy-system optimization models, Unit commitment, Power system modeling, Wind energy integration 
Nomenclature

Abbreviations

$B A T \quad$ Battery storage

$C C G T \quad$ Combined cycle gas turbine

$C O A L S C$ Super-critical coal-fired power plant

$C U C \quad$ Clustered unit commitment

GEP Generation expansion planning

GHG Greenhouse gas

IRES Intermittent renewable energy sources

NUC Nuclear power plant

OCGT Open cycle gas turbine

PHS Pumped hydro storage

$P V \quad$ Photovoltaic

$U C \quad$ Unit commitment

$L P \quad$ Linear programming

$M I L P \quad$ Mixed integer linear programming

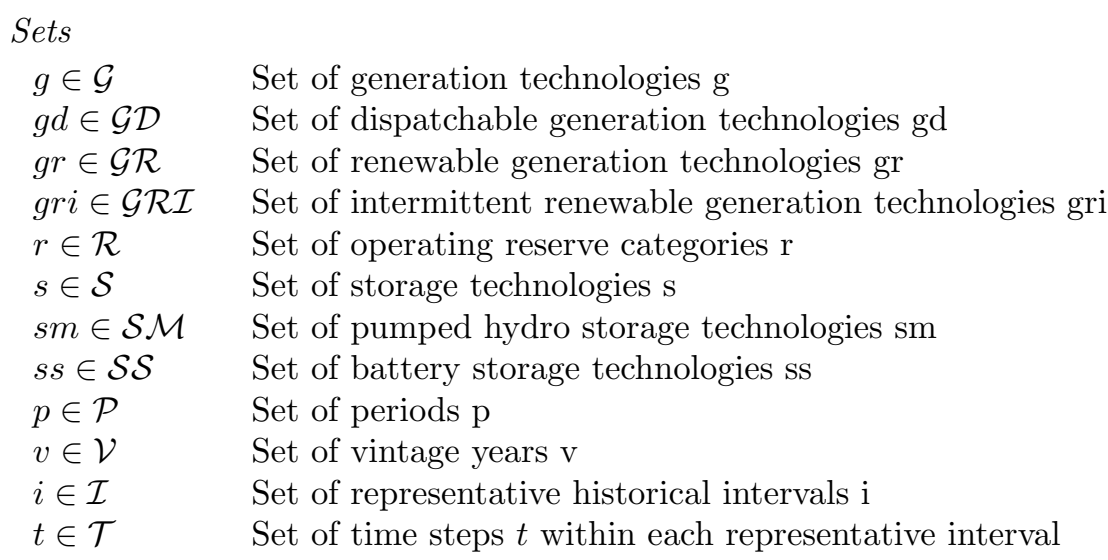

\section{Parameters}

$\bar{D}_{p}$

$D_{p, i, t}$

Annual peak demand for electrical power

$[M W]$

Demand for electrical power

$C_{g, p}^{I N V}$

$\stackrel{M, p}{M} U_{g}^{L T}$

Overnight investment cost

$[€ /(M W)]$

Mark-up factor to account for the lead time for construc-

$L T_{g}$

$S_{g, p}^{F R A C}$

tion

Construction time (lead time)

Fraction of the value salvaged at the end of the time horizon

$T_{g}^{L I F E}$

CFOM

$C^{V / S, p}$

$C_{s, p}^{g / s, v}, E N$

Technical life time

Fixed operations and maintenance cost

Variable operations and maintenance cost

Investment cost for energy reservoir of storage technologies 


\begin{tabular}{|c|c|c|}
\hline$C_{g d, v}^{R A M P}$ & Ramping cost & $\begin{array}{c}{[€ /(\Delta} \\
M W)]\end{array}$ \\
\hline$C_{s, p}^{I N V, C A P}$ & $\begin{array}{l}\text { Investment cost for charging and discharging capacity of } \\
\text { storage technologies }\end{array}$ & {$[€ /(M W)]$} \\
\hline$C_{g d, v}^{S U}$ & Start-up cost & {$[€ /(\Delta)$} \\
\hline$A F_{g d / s, v}$ & Availability factor & {$[-]$} \\
\hline$C P T_{g / s, v, p}$ & $\begin{array}{l}\text { Fraction of the capacity of a certain technology installed in } \\
\text { a certain vintage year which is still operational in period } \\
\text { p }\end{array}$ & {$[-]$} \\
\hline$N C_{g d, v, p}$ & Generation cost at minimum power output & {$[€ / h]$} \\
\hline$M C_{g d, v, p}$ & Marginal fuel and emission cost & {$[€ / M W h]$} \\
\hline $\bar{P}_{g d, v}$ & Maximum operating point & {$[M W]$} \\
\hline$\underline{P}_{g d, v}$ & Minimum operating point & {$[M W]$} \\
\hline$M U T_{g d, v}$ & Minimum up time & {$[h]$} \\
\hline$S U_{g d, v}$ & Maximum power of plant starting up & {$[M W]$} \\
\hline$S D_{g d, v}$ & Maximum power of plant shutting down & {$[M W]$} \\
\hline$S U T_{g d / s m, v}$ & Time required for starting up & {$[h]$} \\
\hline$S D T_{g d / s m, v}$ & Time required for shutting down & {$[h]$} \\
\hline & Round-trip efficiency & {$[-]$} \\
\hline & Ramp rate & {$\left[\% \bar{P}_{g d, v} / h\right]$} \\
\hline$M D T_{g d, v}$ & Minimum down time & {$[h]$} \\
\hline$C F_{g r i, v, p, i, t}$ & Capacity factor & {$[-]$} \\
\hline$T_{p}^{G H G}$ & Tax for greenhouse gas emissions & {$\left[€ / \operatorname{ton}^{C O_{2}^{e q}}\right]$} \\
\hline$P M$ & Planning reserve margin & {$[-]$} \\
\hline$V O L L$ & Penalty for load shedding & {$[€ / M W h]$} \\
\hline VOLR & Penalty for shedding of operating reserves & {$[€ / M W h]$} \\
\hline$S_{g, p}$ & Support for renewable electricity generation & {$[M W]$} \\
\hline$S_{r}^{S P I N}$ & Minimum share of spinning reserves & {$[-]$} \\
\hline$R_{r}^{D E M}$ & Share of demand for which operating reserves are required & {$[-]$} \\
\hline$R_{r, g r i}^{F E}$ & $\begin{array}{l}\text { Share of scheduled intermittent generation for which op- } \\
\text { erating reserves are required }\end{array}$ & {$[-]$} \\
\hline$T_{r}^{D U R}$ & $\begin{array}{l}\text { Duration that storage technologies are required to be able } \\
\text { to provide reserves }\end{array}$ & {$[h]$} \\
\hline$T_{r}^{A C T}$ & Required activation time for operating reserve & {$[h]$} \\
\hline$\underline{D U R}_{s, v}$ & $\begin{array}{l}\text { Minimum energy reservoir capacity relative to rated elec- } \\
\text { trical power }\end{array}$ & {$[h]$} \\
\hline$\overline{D U R}_{s, v}$ & $\begin{array}{l}\text { Maximum energy reservoir capacity relative to rated elec- } \\
\text { trical power }\end{array}$ & {$[h]$} \\
\hline$\underline{P}_{s m, v}^{C}$ & Minimum operating point while charging & {$[M W]$} \\
\hline$\underline{P}_{s m, v}^{D}$ & Minimum operating point while discharging & {$[M W]$} \\
\hline$W_{i}$ & $\begin{array}{l}\text { Number of times representative interval } i \text { is repeated } \\
\text { within one year }\end{array}$ & {$[-]$} \\
\hline & Discount rate & {$[-]$} \\
\hline
\end{tabular}


$\begin{array}{llc}\Delta_{t} & \text { Duration of time step t } & {[h]} \\ E O H & \text { Final year in the modeled time horizon } & {[-]} \\ M Y_{p} & \text { Milestone year corresponding to period p } & {[-]} \\ S Y_{p} & \text { First year corresponding to period p } & {[-]} \\ E Y_{p} & \text { Last year corresponding to period p } & {[-]} \\ y_{0} & \text { Reference year to which all costs are discounted } & {[-]}\end{array}$

Decision Variables

\begin{tabular}{|c|c|c|}
\hline$c_{p}^{v o m}$ & Variable operations and maintenance cost in period $\mathrm{p}$ & {$[€]$} \\
\hline$c_{p}^{s u}$ & Start-up costs in period $\mathrm{p}$ & {$[€]$} \\
\hline$c^{l l}$ & Load-shedding costs in period $\mathrm{p}$ & {$[€]$} \\
\hline$c_{p}^{i r}$ & Reserve-shedding costs in period $\mathrm{p}$ & [€] \\
\hline$v_{n}^{\text {salv }}$ & Salvage value of investments made in period $p$ & {$[€]$} \\
\hline$v_{p}^{\text {res }}$ & Support for renewable electricity generation in period $p$ & {$[€]$} \\
\hline$c_{p}^{f o m}$ & Fixed operations and maintenance cost during period $\mathrm{p}$ & {$[€]$} \\
\hline$c_{p}^{i n v}$ & Investment cost of investments made in period $p$ & {$[€]$} \\
\hline$c_{p}^{g e n}$ & Generation costs in period $\mathrm{p}$ & {$[€]$} \\
\hline$c_{p}^{\text {ramp }}$ & Ramping costs in period $\mathrm{p}$ & {$[€]$} \\
\hline & Number of available units & \\
\hline$n_{g d, v, p, i, t}^{s d}$ & Number of units shutting down & \\
\hline$g_{g d, v, p, i, t}$ & $\begin{array}{l}\text { Electrical power generation above the minimum operating } \\
\text { point }\end{array}$ & I] \\
\hline$r a m p_{g d, v, p, i, t}$ & Change in electrical power output & {$[W]$} \\
\hline$n_{g d, v, p, i, t}^{o n}$ & Number of online units & \\
\hline$n_{g d, v, p, i, t}^{s u}$ & Number of units starting up & $f$ \\
\hline$c a p_{g, v}^{n e w}$ & Capacity installed in period $\mathrm{v}$ & {$[M V$} \\
\hline$c a p_{g d, v, p}^{a v}$ & $\begin{array}{l}\text { Capacity installed in period } v \text { that is available in period } \\
\mathrm{p}\end{array}$ & {$[M W]$} \\
\hline$g_{e n} n_{g, v, p, i, t}$ & $\begin{array}{l}\text { Electrical power generation of a technology g installed in } \\
\text { period } v \text { during time interval }(\mathrm{p}, \mathrm{i}, \mathrm{t})\end{array}$ & {$[M W]$} \\
\hline$l l_{p, i, t}$ & Unserved demand & {$[M W]$} \\
\hline curt $_{g r i, v, p, i, t}$ & Curtailment of renewable electricity generation & \\
\hline$y_{g r i, v, p, i, t}$ & $\begin{array}{l}\text { Binary variable indicating whether the IRES generation } \\
\text { level is below the level that can be guaranteed with a } \\
\text { reasonable certainty }\end{array}$ & {$[\mathrm{l}$} \\
\hline$z_{g r i, v, p, i, t}$ & $\begin{array}{l}\text { Binary variable indicating whether IRES can provide up- } \\
\text { ward reserves }\end{array}$ & [1 \\
\hline gen $n_{\text {gri } i, v, p, i, t}^{\text {uncertain }}$ & $\begin{array}{l}\text { IRES generation above the value that can be guaranteed } \\
\text { with a reasonable certainty }\end{array}$ & [] \\
\hline gen $_{\text {gri } i, v, p, i, t}^{\text {certain }}$ & $\begin{array}{l}\text { IRES generation that can be guaranteed with a reasonable } \\
\text { certainty }\end{array}$ & {$[\mathrm{l}$} \\
\hline$r_{r, g d, v, p,}^{+, s p i n}$ & Spinning upward reserves procured & {$[M W]$} \\
\hline & Upward reserves procured & {$[M W]$} \\
\hline$r_{r, g d, v, p, i, t}^{+, n s}$ & Non-spinning upward reserves procured & \\
\hline
\end{tabular}


$n_{g d, v, p, i, t}^{+, n s}$

$l r_{r, p, i, t}$

$e_{s, v, p, i, t}^{l}$

$p_{s, v, p, i, t}^{c}$

$e_{s, v, p, i, t}^{f}$

$p_{s, v, p, i, t}^{d}$

$\operatorname{cap}_{s, v, p}^{a v}$

$\operatorname{cap}_{s, v}^{n e w}$

$\operatorname{cap}_{s, v}^{n e w,}$

$n^{d, s u}$

$n_{s m, v, p, i, t}^{d, s u}$

$n^{d, o n}$

$n_{s m, v, p, i, t}$

$n^{d, a v}$

$n_{s m, v, p}$

$n_{s m, v, p, i, t}$

$n_{\text {d,sd }}$

$n_{c, o n, p, i, t}$

$n_{s m, v, p, i, t}^{c, o n}$

$n_{s m}^{c, a v}$

$n_{s, s u}^{c, v, p}$

$n_{s m, v, p, i, t}^{c, s u}$

$r_{r, s, v, p, i, t}^{+}$

$r, s, v, p, i, t$
,+ spin,$c$

$r_{r, s m, v, p, i, t}^{+, s p i n, c}$

$r_{r, s, v, p, i, t}^{+, d}$

$r_{r, s, v, p, i, t}^{+, c}$

$r_{r, s m, v, p, i, t}^{+, s p i n, d}$

$, v, p, i$,

$n_{s m, v, p, i, t}^{+, s u, d}$

$r_{r, n s, c}^{+, n, v, i,}$

$r_{r, s m, v, p, i, t}$

$r^{+, n s, d}$

$r, s m, v, p, i, t$

$n_{s m, v, p, i, t}^{+, s d, c}$
Number of units procured to start-up for providing upward reserves

Curtailed operating reserves

Energy content of storage at in the last repetition of the representative interval

Electrical power consumption while charging

Energy content of storage at in the first repetition of the representative interval

Electrical power output while discharging

Available charging/discharging power capacity

Installed charging/discharging power capacity

Installed energy reservoir capacity

Number of units starting up to discharge

Number of online discharging units

Number of available units for discharging

Number of charging units shutting down

Number of discharging units shutting down

Number of online charging units

Number of available units for charging

Number of units starting up to charge

Upward reserves procured from storage technologies

Spinning upward reserves procured from pumped hydro storage technologies while charging

Upward reserves procured from storage technologies by adapting discharging output

Upward reserves procured from storage technologies by adapting charging output

Spinning upward reserves procured from pumped hydro storage technologies while discharging

Number of units procured to start-up discharging for providing upward reserves

Non-spinning upward reserves procured from pumped hydro storage technologies by shutting down pumping units Non-spinning upward reserves procured from pumped hydro storage technologies by starting up turbining units Number of units procured to shut down charging for providing upward reserves
$[M W]$

\section{Introduction}

Bottom-up, long-term energy system planning models or generation expansion planning (GEP) models are frequently used to analyze pathways for the transition of the energy/electrical power system and to deduce policy advice. Due to computational restrictions, these models typically use a limited level of temporal detail, and do not integrate detailed operational constraints [1, 2]. More specifically, the technical constraints 
faced by individual power plants (e.g., the minimum operating point, ramp-rate restrictions, start costs, etc.), as well as detailed system constraints aiming to ensure reliability (e.g., operating-reserve requirements) are typically not considered. This level of detail has historically been reserved for operational models, such as unit commitment (UC) models. However, in the context of an increasing penetration of intermittent renewable energy sources (IRES), concerns have recently been voiced that neglecting this level of detail in planning models might yield infeasible or sub-optimal solutions to the planning problem.

These concerns regarding flexibility in power systems with high penetration levels of IRES have spurred multiple authors to integrate detailed flexibility constraints in GEP models. Kirschen, Ma, Silva and Belhomme [3, Ma, Silva, Belhomme, Kirschen and Ochoa 4 and de Sisternes [5] have extended a UC model to consider investment decisions in individual plants. Similarly, Koltsaklis and Georgiadis [6] integrate UC constraints in a multi-period and multi-regional GEP model and apply it to the Greek power system. Pereira, Ferreira and Vaz [7] develop a mixed integer non-linear multi-period GEP model with integrated UC constraints and detailed fuel cost curves and apply it to the Portuguese system. To maintain computational tractability, the different authors all make use of a number of representative weeks or days to represent an entire year. Recently, a number of methods have been developed to carefully select representative sets of weeks or days. These methods are either based on enumeration (see e.g., [8, clustering algorithms (see e.g., [9]) or dedicated optimization models (see e.g., [10]). A different strategy to reduce the computational burden, as proposed by Flores-Quinoz, Palma-Behnke, Zakeri and Moreno [11, is to use decomposition techniques. Other authors have instead focused on altering the formulation of the UC constraints. For instance, Palmintier and Webster [12, 13] have developed a clustered unit commitment (CUC) formulation, in which the binary commitment variables for individual generation units are replaced by an integer commitment variable per cluster of identical or similar generation units. This allows reducing the combinatorial state space, the number of variables and the number of constraints, and is shown to significantly reduce the computational cost. Palmintier [14] and Zhang, Capuder and Mancarella [15] explored using continuous rather than integer commitment variables to further reduce the computational burden and showed that this has a limited impact in terms of accuracy. Hua, Baldick and Wang [16 integrate a convex relaxation of the UC problem, and show that this method outperforms the clustering method in terms of accuracy. An additional advantage of this methodology is that transmission constraints can easily be integrated. Other authors have used more stylized representations of the UC constraints. For instance, Welsch et al. [17 have extended the OSeMOSYS energy system optimization model to account for operating reserve requirements, but do not consider start-up costs. In both the LIMES-EU power-system optimization model and the ReEDS model, the online/committed capacity cannot change between certain periods, and the power output is restricted by a minimal loading requirement. In addition, simplified constraints for operating reserves are incorporated in the ReEDS model. De Jonghe, Delarue, Belmans and D'haeseleer [18] introduce simplified ramping constraints to represent the limited flexibility of thermal power plants. Finally, Batlle and Rodilla [19] and Staffell and Green [20, have incorporated flexibility aspects in analytical screening curve models. For a more detailed overview of the different methods developed in the literature, we refer to Collins et al. 21, Koltsaklis and Dagoumas [2] 
and Chapter 5 of $[22]$.

Whereas a myriad of different approaches has been developed to integrate UC constraints in GEP models, fewer authors have investigated the actual impact of not incorporating UC constraints. By taking the capacity mix resulting from a GEP model which does not incorporate UC constraints and reevaluating the dispatch decisions using a UC model, Deane, Chiodi, Gargiulo and Ó Gallachóir [23] and Poncelet et al. 24] both conclude that neglecting these technical constraints leads to an overestimation of the value of baseload and IRES technologies whereas flexible technologies are not sufficiently valued. In turn, this was shown to lead to an underestimation of the operational costs, and hence the total system cost. Similar findings were found by Belderbos and Delarue [25. Shortt, Kiviluoma and O'Malley [26] have investigated the impact of UC constraints for a broad set of test systems differing in load and renewable time series, the penetration level of wind energy and the type of generators using a UC model. They highlight that the impact of neglecting UC constraints is highly system specific, but generally becomes higher with an increasing penetration of IRES. Additionally, Palmintier and Webster [12, 27. emphasize that the impact of UC constraints not only tends to increase with an increasing penetration of IRES, but also tends to grow as more stringent carbon policies are imposed. In addition, Palmintier [14, as well as Poncelet 22 have analyzed the impact of individual UC constraints on the results. Both Palmintier and Poncelet came to the conclusion that operating reserve requirements play an important role, while certain other constraints, such as hourly ramping constraints do not. Palmintier and Webster [12, 27. further developed a clustered UC formulation which allowed them to directly investigate the impact of neglecting the UC constraints in the investment planning problem. Also they conclude that neglecting UC constraints has a significant impact on the capacity mix deemed optimal by the model and the resulting generation mix, and hence cannot be ignored. Similar results are again found by Abdin and Zio [28] using a similar methodology. Using a GEP model with an integrated convex relaxation of the UC problem and a test case based on the ERCOT system, Hua, Baldick and Wang [16] similarly find that neglecting flexibility aspects in GEP models result in suboptimally low investments in flexible generators, leading to reserve shortage, load shedding and additional renewable curtailment. Nogales, Wogrin and Centeno 29 integrate UC constraints in a generation expansion equilibrium problem for oligopolistic markets. By comparing the results to those of an identical model without the UC constraints, they find that not incorporating UC constraints leads to lower investments in flexible peak-load technologies and an underestimation of the total system costs.

In summary, the recent literature has assessed the impact of UC constraints on the GEP problem. Under the assumptions taken, neglecting UC constraints was shown to have a highly significant impact in terms of the capacity mix, the generation mix, carbon emissions and the cost projections, particularly for highly renewable or carbon constrained electricity systems. As such, the conclusion from this strand of literature is that UC constraints should not be neglected when planning towards a highly renewable or carbon constrained electricity system. Recently, the focus of the literature has shifted towards developing methods to tractably integrate these UC constraints in planning models. 
However, the assessment of the impact of integrating UC constraints in planning models is not only dependent on the system composition (i.e., the share of renewables and the degree of decarbonization), but also depends strongly on certain assumptions made regarding the availability of flexibility as well as the need for flexibility in future power systems. In this context, the existing literature can further be complemented since in the current literature:

- thermal generators are frequently considered the only source of flexibility (aside from curtailment of IRES electricity generation), particularly in the literature assessing the impact of UC constraints (see e.g., 25, 12, 27, 28, 16, 29] ) $)^{1}$. As will be shown in the remainder of this paper, relaxing this assumption drastically reduces the impact of neglecting UC constraints and hence significantly changes some of the conclusions drawn in the current literature;

- the sensitivity to certain input data and specific modeling assumptions that need to be taken when integrating UC constraints in GEP models have not been investigated. More specifically, assumptions need to be taken regarding: (i) the current and future cycling capabilities of thermal power plants and (ii) the amount and type of operating reserves that will be required in future power systems. Regarding the cycling capabilities of thermal power plants, a wide range of values is reported in the literature (see e.g., [30, 31, 32]). Regarding the characterization of operating-reserve requirements, rules of thumb based on current practices or simple extrapolations are regularly used. More details on these assumptions are presented in Section 2.2.2. As will be shown, the assumptions taken here are of a higher or similar importance than the level of detail with which operational UC constraints are represented in the GEP model.

The goal of this paper is threefold. The first goal is to revisit the impact of UC constraints in GEP models for varying assumptions regarding the availability of other sources of flexibility, the cycling capabilities of thermal generators and the need for operating reserves. In this setting, the second goal is to identify when it is relevant to consider UC constraints. The third and final goal is to gain insights into how accurate the UC constraints should be formulated. In this regard, the errors induced by modeling the UC constraints in a varying level of detail are contrasted to the sensitivity of the model results to certain assumptions taken when integrating UC constraints.

This paper contributes to the existing literature by:

1. showing that the impact of integrating UC constraints in planning models is limited, and can be significantly overestimated if alternative sources of flexibility (aside from

\footnotetext{
${ }^{1}$ An exception is the work of Shortt, Kiviluoma and O'Malley [26] which considers a scenario in which run-of-river hydro coupled to a reservoir is considered. However, as will be discussed in Section 5.2 one of the main benefits of alternative sources of flexibility, such as batteries, pumped-hydro storage (PHS) and active demand response (ADR), is their ability to provide upward reserves without having to generate electricity. As such, these technologies can avoid the need to keep generators spinning at minimum generation level during periods of high renewable electricity generation. As a result, these alternative sources of flexibility allow absorbing more renewable electricity generation. The flexibility offered by batteries, PHS and ADR is hence fundamentally different than the flexibility offered by run-of-river hydro coupled to a reservoir that can rather be seen as a flexible source of baseload generation.
} 
cycling of thermal generators and curtailment of IRES) are not considered. As such, this paper strongly nuances the conclusions regarding the impact of incorporating UC constraints drawn based on existing studies in which alternative sources of flexibility are not considered.

2. showing that integrating UC constraints in GEP models is not sufficient to achieve qualitative results. Specifically, it is shown that it is imperative to incorporate alternative sources of flexibility, such as storage technologies and active demand response. As will be shown in the remainder of the paper, integrating UC constraints without explicitly considering alternative sources of flexibility can lead to errors in cost projections and biases in investment decisions which can be significantly higher than the impact of simply neglecting UC constraints;

3. indicating when it is relevant to consider UC constraints in GEP models. As will be shown in the remainder of the paper, incorporating UC constraints is mainly relevant if one specifically aims to investigate the role of dedicated flexibility providers (e.g., batteries);

4. exposing the high sensitivity of GEP model results to the assumptions made regarding the characterization of operating reserves and, in particular, the assumed cycling capabilities of thermal generators. As such, we show that integrating UC constraints in GEP models is not sufficient to get robust results and recommend modelers to explore these sensitivities. In addition, we point to specific modeling challenges that need to be addressed in future research;

5. showing that the approximation errors of existing computationally lean formulations to integrate UC constraints in planning models are an order of magnitude smaller or of a similar order of magnitude than the sensitivity to the assumptions made regarding the availability of alternative flexibility providers, the flexibility that can be provided by current and future thermal generators, and the future need for operating reserve requirements respectively.

It must be noted that this paper does not present a novel methodology to integrate UC constraints in GEP models. Rather, this paper explores the impact of certain assumptions that are typically made/need to be made when integrating UC constraints in GEP models, leading to new insights that are key for both users of GEP models and researchers investigating or developing novel methods to integrate UC constraints in GEP models.

The remainder of this paper is organized as follows. First, Section 2 presents the methodology used to assess the impact of neglecting detailed technical constraints for a variety of assumptions and to assess the sensitivity to these assumptions. Next, Section 3 provides a detailed mathematical formulation of the models used in this paper. Section 4 subsequently describes the case study. The results are presented and discussed in Section 5. Finally, the main conclusions are summarized in Section 6 .

\section{Methodology}

To assess the impact of not incorporating unit commitment (UC) constraints, the results of a generation expansion planning (GEP) model with integrated UC constraints are compared to those of a traditional GEP model that does not integrate UC constraints. 
The results of both models are compared in terms of the projected total annual system cost, the capacity mix, the generation mix and the $\mathrm{CO}_{2}$ emissions for two scenarios with high shares of variable renewable energy sources but differing in the carbon price.

We complement the existing literature by assessing the impact of integrating UC constraints in GEP model for a number of sensitivity cases varying in the consideration of other flexibility providers, the assumed flexibility of thermal generators, and the assumptions taken for characterizing operating reserve requirements. As such, we can analyze to what extent the conclusions with regard to the importance of integrating UC constraints in GEP models change when alternative flexibility providers are considered. In addition, the sensitivity to certain modeling assumptions can be quantified. Finally, these sensitivities are compared to the errors introduced by using an approximate but computationally lean formulation of the unit commitment constraints.

The different models used in the paper are first presented in Section 2.1. Next, the different sensitivity cases are first presented in detail in Section 2.2 .

\subsection{Model versions}

The model used in this paper is the LUSYM generation expansion planning model that co-optimizes investments in thermal generators, IRES and storage technologies. Three versions of this model are considered:

- MIP: generation expansion planning model with integrated clustered unit commitment (CUC) model. This CUC model groups together identical power plants and replaces the binary commitment variables for individual generators by an integer variable per technology cluster. The following elements are accounted for in the model: ramping constraints, the minimum operating point, minimum up and down times, start-up costs, part-load efficiency losses and operating reserve requirements. In earlier work, the CUC model has been validated by comparing it with a traditional UC model employing binary variables. The errors introduced by clustering identical power plants were shown to be negligible 33 .

- LP: generation expansion planning model identical to the MIP model, but uses continuous rather than integer commitment variables.

- MO: traditional generation expansion planning model that does not integrate unit commitment constraints. Without these constraints, generators will be dispatched according to the merit-order list. We therefore refer to this model as the MO model.

The mathematical formulation of the models used in this paper is described in Section 3.

\subsection{Sensitivity cases}

2.2.1. Sensitivity cases related to alternative flexibility providers and cycling capabilities

Four different sensitivity cases are considered differing in the availability of alternative flexibility providers and the assumed cycling capabilities of thermal generators (i.e., the supply side for flexibility). An overview of the 4 considered sensitivity cases on the supply of flexibility is presented in Tab. 4 


\begin{tabular}{lcccc}
\hline Case & No stor & No stor & Stor & Stor \\
& Inflex & Flex & Inflex & Flex \\
\hline Storage available & no & no & yes & yes \\
Flexibility of thermal generators & low & high & low & high \\
\hline
\end{tabular}

Table 4: Overview of the sensitivity cases with respect to the consideration of alternative flexibility providers and the cycling capabilities of thermal generators.

First, regarding the availability of alternative flexibility providers, we consider a case with or without the opportunity to invest in electricity storage-technology types. Two types of storage-technology types are considered: pumped-hydro storage (PHS) and battery storage (BAT). The cycling characteristics of both storage technologies are presented in Tab. 5. Cases in which investments in storage-technology types are and are not considered are indicated by a 'Stor' and 'No_stor' respectively.

\begin{tabular}{lll}
\hline Technical characteristic & PHS & BAT \\
\hline MSOP while charging $\left[\% / P_{\text {nom }}\right]$ & 60 & 0 \\
MSOP while discharging $\left[\% / P_{\text {nom }}\right]$ & 30 & 0 \\
Ramp rate [\% $P_{\text {nom }} /$ min] & 20 & 100 \\
Start-up time charging [min] & 15 & 0 \\
Start-up time discharging [min] & 5 & 0 \\
Shut-down time charging [min] & 5 & 0 \\
Shut-down time discharging [min] & 10 & 0 \\
\hline
\end{tabular}

Table 5: Cycling characteristics of the energy storage technologies. Data is taken from 34, 30.

Second, as discussed in 31, there is a large range of data regarding the cycling characteristics of thermal power plants. In this paper, we consider two sets of cycling characteristics. In the first set, the flexibility of thermal power plants is near the lower limit of the ranges reported in the literature (referred to as the 'Inflex' case) whereas in the second set, the flexibility of thermal power plants is assumed to be near the upper limit of the ranges specified in the literature (referred to as the 'Flex' case). The cycling characteristics adopted in both cases are presented in Tab. 6. The range of cycling capabilities of thermal power plants is adopted from [31, 32, 30].

\subsubsection{Sensitivity cases related to operating reserve requirements}

Tab. 7 presents an overview of how reserve requirements are characterized in a number of state-of-the-art GEP models. From this table, it can be observed that between different models, significant differences exist in terms of the sizing of reserve requirements and the required activation times (and whether or not fast-starting units can provide reserves).

In addition to these differences in terms of the required amount of reserve requirements and the required activation times, it can be observed that the methodologies used to endogenously size reserves in most GEP models are to some extent simplified, and could be further refined in following ways. 


\begin{tabular}{llllll}
\hline Technical characteristic & Case & NUC & COAL SC & CCGT & OCGT \\
\hline Minimum operating & Inflex & 50 & 40 & 50 & 50 \\
point $\left[\% / P_{\text {nom }}\right]$ & Flex & 40 & 25 & 30 & 20 \\
\hline Eff. loss at minimum & Inflex & 5 & 2 & 11 & 22 \\
operating point $[\%$ pt $]$ & Flex & 1.8 & 2 & 3.2 & 9 \\
\hline Ramp rate & Inflex & 0.25 & 0.66 & 0.83 & 0.83 \\
{$\left[\% P_{\text {nom }} /\right.$ min $]$} & Flex & 5 & 4 & 10 & 25 \\
\hline Minimum up & Inflex & 24 & 10 & 6 & 1 \\
time $[\mathrm{h}]$ & Flex & 6 & 6 & 1 & 0 \\
\hline Minimum down & Inflex & 24 & 10 & 6 & 1 \\
time $[\mathrm{h}]$ & Flex & 4 & 3 & 1 & 0 \\
\hline Start-up energy & Inflex & 46.7 & 3.6 & 1.8 & 0.0 \\
{$\left[M W h_{t h} / \Delta M W_{e}\right]$} & Flex & 16.7 & 3.6 & 1.5 & 0.0 \\
\hline Start-up depreciation & Inflex & 1.7 & 70.3 & 68.4 & 105.0 \\
{$\left[\right.$ EUR $\left./ \Delta M W_{e}\right]$} & Flex & 1.7 & 45.1 & 24.5 & 19.4 \\
\hline Start-up time & Inflex & 50 & 8 & 1 & 0.33 \\
{$[\mathrm{~h}]$} & Flex & 24 & 2 & 1 & 0.17 \\
\hline
\end{tabular}

Table 6: Cycling characteristics of thermal generators

First, different sources of uncertainty (e.g., demand forecast errors, wind generation forecast errors and solar generation forecast errors) are often treated independently, i.e., the total required reserves are determined by summing up the reserves required to deal with individual sources of uncertainty. This can lead to an overestimation of the required reserves.

Second, the required reserves to deal with IRES forecast errors are typically assumed to increase linearly with their instantaneous power generation (see Tab. 7). Whereas this linear relationship might accurately reflect the need for operating reserves in systems with a low penetration of IRES, it can significantly overestimate the need for operating reserves in systems with a high penetration of IRES where there could be regular periods with scheduled curtailment. Indeed, if there is scheduled curtailment, this scheduled curtailment directly reduces the exposure to forecast errors and hence the need for operating reserves. In the reserve sizing rules that are currently typically applied in GEP models, the reduction of the exposure to forecast errors when there is scheduled curtailment is not properly taken into account.

For instance, in NREL's RPM, reserves to deal with forecast errors of wind generation are dimensioned as $10 \%$ of the wind generation. Assuming now a period during which the forecasted wind generation equals 10,000 MW, of which 2,000 MW is scheduled to be curtailed, the reserve requirement in the RPM equals $10 \%$ of the scheduled wind generation, or thus $800 \mathrm{MW}$. However, in such cases, the worst case would be that the wind power that could be generated is $10 \%$ below the forecasted value, meaning that 


\begin{tabular}{|c|c|c|}
\hline Model & Activation time & Sizing \\
\hline \multirow[t]{3}{*}{ RPM 35. } & $\begin{array}{l}\text { Sub } 5 \text { minutes, } \\
100 \% \text { spin }\end{array}$ & $1 \%$ of demand \\
\hline & $\begin{array}{l}10 \text { minutes, } 50 \% \\
\text { spin }\end{array}$ & $\begin{array}{l}\text { Maximum of } 6 \% \text { of demand and the largest } \\
\text { contingency }\end{array}$ \\
\hline & 1 hour, $100 \%$ spin & $\begin{array}{l}10 \% \text { of wind generation }+7.5 \% \text { of solar } \\
\text { generation }\end{array}$ \\
\hline \multirow{3}{*}{ ReEDS 36] } & $\begin{array}{l}\text { Sub-minute, } 100 \% \\
\text { spin }\end{array}$ & $1.5 \%$ of demand \\
\hline & $\begin{array}{l}10 \text { minutes, } 50 \% \\
\text { spin }\end{array}$ & $6 \%$ of demand \\
\hline & $\begin{array}{l}\text { roughly an hour, } \\
17 \% \text { spin }\end{array}$ & $\begin{array}{l}\text { Maximum difference in generation output } \\
\text { between } 2 \text { consecutive hours in the last } 15 \\
\text { days }\end{array}$ \\
\hline \multirow{2}{*}{$\begin{array}{l}\text { Palmintier and } \\
\text { Webster }[12]\end{array}$} & 5 minutes & $\begin{array}{l}1 \% \text { of demand }+0.385 \% \text { of installed wind } \\
\text { capacity }\end{array}$ \\
\hline & $\begin{array}{l}10 \text { minutes, } 50 \% \\
\text { spin }\end{array}$ & $\begin{array}{l}\text { Maximum of two largest generators and } 3.3 \% \\
\text { of demand }+7.95 \% \text { of installed wind capacity } \\
+13.9 \% \text { of instantaneous wind generation }\end{array}$ \\
\hline
\end{tabular}

Table 7: Overview of the reserve requirements adopted in different GEP models

1,000 MW of potential wind power generation would be uncertain. However, due to the fact that here is scheduled curtailment of 2,000 MW, in case of lower than predicted wind speeds, it would be perfectly feasible to activate the curtailed wind power. As such, it can be considered that the scheduled curtailment directly reduces the exposure to forecast errors that need to be covered by thermal generators or other flexibility providers. This is visualized in Fig. 1 .

In addition, if the scheduled generation from IRES is below a certain amount that can be guaranteed with a reasonable certainty, IRES could even provide upward reserves to deal with other types of uncertainty (e.g., demand forecast errors). Further note that, as in most GEP models, only upward reserves are considered. This because ensuring sufficient downward reserves tends to be rather inexpensive 35. We assume that this remains the case in future power systems. This because, in contrast to the provision of upward reserves by IRES, downward reserves could relatively inexpensively be provided by IRES. Therefore, in this paper, downward reserves are also not considered.

In the simulations presented in this paper, the reference model (REF) adopts the reserve requirements from NREL's Resource Planning Model (RPM) 35], as presented in Tab. 7. To assess the sensitivity to the assumptions regarding the requirements for operating reserves, three additional cases are considered. A first case does integrate all technical constraints of individual generators but does not impose operating reserve requirements (referred to as the 'No_op_res' case). A second additional case has $20 \%$ lower reserve requirements as the REF case (referred to as the 'REF-20\% case). A final case does impose reserve requirements, but does take into account that scheduled 


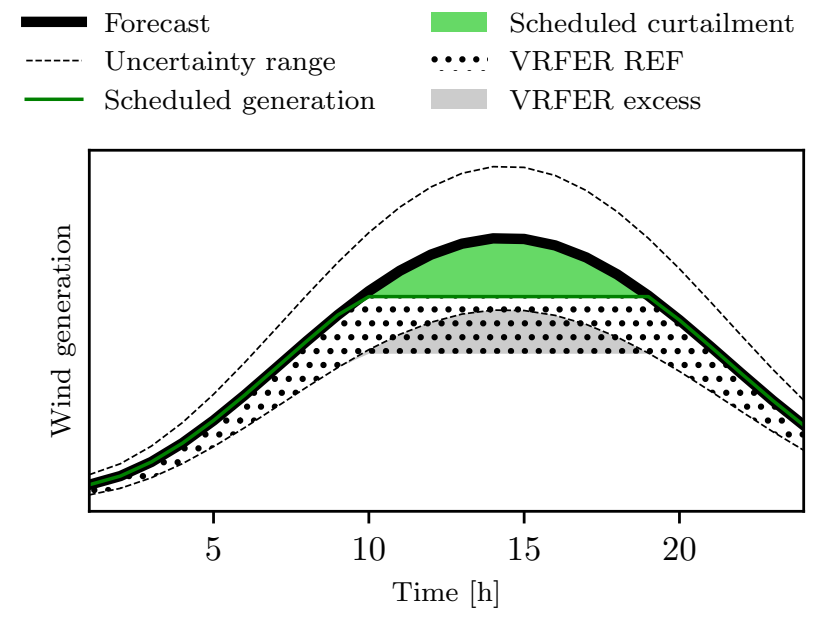

Figure 1: Illustration of the sizing of variable renewable forecast error reserve (VRFER) requirements in generation expansion planning (GEP) models. The dotted area indicates the VRFER requirements typically imposed in current GEP models. The grey area indicates the excess of VRFER requirements if there is scheduled curtailment.

curtailment reduces the need for reserves to deal with IRES forecast errors, i.e., the reserve requirements are formulated to not consider the grey areas in Fig. 1] (referred to as the 'Curt' case). An overview of the considered cases is presented in Tab. 8 .

\begin{tabular}{ll}
\hline Case & Description \\
\hline REF & Reserve requirements adopted from NREL's RPM \\
Curt & Improved accounting of curtailment and the ability of \\
& IRES to provide upward reserves. \\
REF-20\% & 20\% lower reserve requirements than in the REF case \\
No_op_res & No operating reserve requirements considered \\
\hline
\end{tabular}

Table 8: Overview of the sensitivity cases with respect to the characterization of future operating reserve requirements.

\section{Model formulation of the LUSYM GEP model}

This section presents the mathematical formulation of the LUSYM generation expansion planning model that co-optimizes investments in thermal generators, IRES and storage technologies. In the below mathematical formulation, all variables are continuous and nonnegative unless explicitly mentioned.

\subsection{Temporal representation}

In the model, the considered time horizon (e.g., 2018-2050) is divided into a number of periods $p \in \mathcal{P}$, each representing a single or multiple years. During each period, new capacity can be installed. 
In terms of the operation of the electrical power system, each period is represented by a single year, the so-called 'milestone year' (MY). This milestone year is taken as the middle year of the period $p$. To account for the variability of the electricity demand and intermittent renewable energy sources (IRES), the milestone year is disaggregated into a number of representative intervals $i \in \mathcal{I}$ (e.g., days or weeks), each consisting of a number of time steps $t \in \mathcal{T}$ (e.g., hours). This is visualized in Fig. 2 .

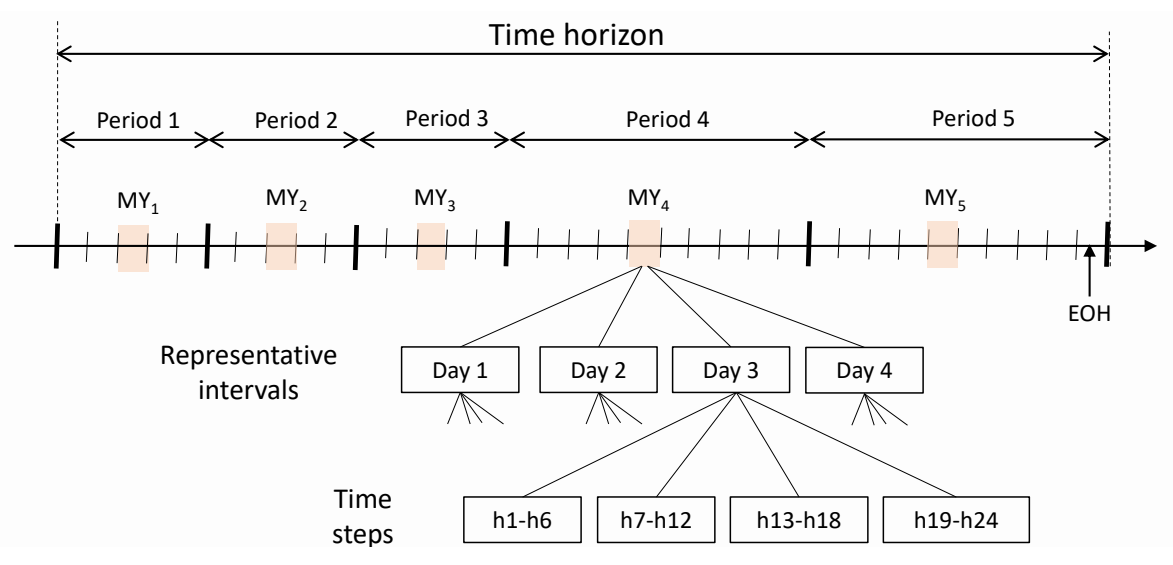

Figure 2: Illustration of the temporal representation in the LUSYM Invest model. The time horizon, the number of periods and their duration, the number of representative intervals and their length (e.g., days or weeks) as well as the resolution of the time steps can be chosen freely.

Within each period, the costs of operating the system are assumed to be equal to the operating costs in the milestone year. This implies that all exogenously-defined, time-dependent, parameters (e.g., fuel prices, electricity demand, etc.) within an entire period are assumed to be equal to the values corresponding to the milestone year of that period.

An exception relates to the technological characteristics (e.g., overnight investment costs, efficiencies, etc.) which are assumed to correspond to the first year in which the plant effectively becomes operational (i.e., the so-called vintage year $v$ ), rather than the milestone year of the period in which the new capacity is installed.

To account for the lead time of new investments, an investment trajectory is assumed such that the newly installed capacity in a period $p$ is assumed to become operational at the first year corresponding to that period. Depending on the construction time, this implies that the construction and the related costs need to be made during a number of years prior to the first year of the period. It is further assumed that the overnight investment cost is divided equally over this construction time.

\subsection{Objective function}

The objective function to minimize is the total discounted system cost. This total discounted system cost consists of investment costs, fixed operations and maintenance 
(FOM) costs, fuel (and emission) related generation costs, variable operations and maintenance (VOM) costs, start-up costs, ramping costs, load-shedding costs and reserveshedding costs. The value of the constructed plants which are still operational after the end of the modeled time horizon (i.e., the so-called 'salvage value') is accounted for via a negative term in the objective function. In addition, possible support mechanisms for renewable electricity generation are also implemented via a negative cost term in the objective function ${ }^{2}$

$\min \sum_{p \in \mathcal{P}}\left[\frac{1}{(1+r)^{\left(M Y_{p}-y_{0}\right)}}\left(c_{p}^{i n v}+c_{p}^{f o m}+c_{p}^{g e n}+c_{p}^{v o m}+c_{p}^{s u}+c_{p}^{r a m p}+c_{p}^{l l}+c_{p}^{l r}-v_{p}^{s a l v}-v_{p}^{r e s}\right)\right]$.

Here, $r$ is the discount rate, $M Y_{p}$ represents the milestone year corresponding to period $p$, and $y_{0}$ is a reference year to which all costs are discounted.

Note that all terms in Eq. (1) represent equivalent lump-sum costs occurring in the milestone year. These cost terms are equivalent to the corresponding costs incurred over the entire period.

\subsubsection{Investment-related costs}

The equivalent lump-sum investment cost for each period $p$ is directly related to the investments in generation technology-types $g \in \mathcal{G}$ and storage technology-types $s \in \mathcal{S}^{3}$.

$$
\begin{aligned}
c_{p}^{i n v}= & \frac{1}{(1+r)^{\left(S Y_{p}-M Y_{p}\right)}} \sum_{g \in \mathcal{G}}\left(\operatorname{cap}_{g, p}^{n e w} C_{g, p}^{I N V} M U_{g}^{L T}\right) \\
& +\frac{1}{(1+r)^{\left(S Y_{p}-M Y_{p}\right)}} \sum_{s \in \mathcal{S}}\left(\left(\operatorname{cap}_{s, p}^{n e w} C_{s, p}^{I N V, C A P}+c a p_{s, p}^{n e w, e} C_{s, p}^{I N V, E N}\right) M U_{s}^{L T}\right)
\end{aligned}
$$

Here, $M U_{g / s}^{L T}$ represents a mark-up factor over the overnight investment $\operatorname{costs} C_{g, p}^{I N V} / C_{s, p}^{I N V, C A P} / C_{s, p}^{I N V, E N}$ to account for the interests during construction. Under the assumption that construction costs are divided equally over the construction period, this mark-up factor is as follows:

$$
M U_{g / s}^{L T}=\frac{\sum_{a=1}^{L T_{g / s}}(1+r)^{a}}{L T_{g / s}}
$$

In addition, the term $\frac{1}{(1+r)^{\left(S Y_{p}-M Y_{p}\right)}}$ is used to convert the resulting cost in the first year of period $p\left(S Y_{p}\right)$ to an equivalent lump-sum cost in the milestone year of that period $\left(M Y_{p}\right)^{4}$

\footnotetext{
${ }^{2}$ From a system perspective, this can be interpreted as there being a constant societal value for renewable electricity generation. From a market perspective, this can be interpreted as a direct subsidy for renewable electricity generation.

${ }^{3}$ The investment variables $\left(\operatorname{cap}_{g, p}^{n e w} / \operatorname{cap}_{s, p}^{n e w} / \operatorname{cap} p_{s, p}^{n e w, e}\right)$ are presented here with one index referring to the set of generation/storage technologies and one index referring to the period in which the investment is made. In the remainder of this text, these variables will be presented more generally as $\operatorname{cap}_{g, v}^{n e w} / \operatorname{cap}_{s, v}^{n e w} / \operatorname{cap}_{s, v}^{n e w, e}$, i.e., with an index $\mathrm{v}$ instead of the index p. This to make a clear distinction between the considered period (p), and the period in which the investment in a certain plant is made (v). For simplicity of notation, the investment variables are presented in Eq. 2 using an index p.

${ }^{4}$ Recall from Section 3.1 that newly installed capacity becomes operational in the first year of the period
} 
The salvage value of the plants becoming operational in period $p$ is a fraction $S_{g / s, p}^{F R A C}$ of the total value of the overall investment where the total value is assumed to be equal to the investment cost.

$$
\begin{aligned}
v_{p}^{\text {salv }}= & \frac{1}{(1+r)^{\left(S Y_{p}-M Y_{p}\right)}} \sum_{g \in \mathcal{G}}\left(S_{g, p}^{F R A C} \operatorname{cap}_{g, p}^{n e w} C_{g, p}^{I N V} M U_{g}^{L T}\right) \\
& +\frac{1}{(1+r)^{\left(S Y_{p}-M Y_{p}\right)}} \sum_{s \in \mathcal{S}}\left(\left(S_{s, p}^{F R A C} c a p_{s, p}^{n e w} C_{s, p}^{I N V, C A P}+S_{s, p}^{F R A C} c a p_{s, p}^{n e w, e} C_{s, p}^{I N V, E N}\right) M U_{s}^{L T}\right)
\end{aligned}
$$

To determine the fraction of the overall value at the end of the modeled time horizon, assumptions need to be made regarding how the value of the asset evolves over its lifetime. Here, similar to most investment planning models (see e.g., the TIMES model [37]), it is assumed that the value is constant throughout the asset's technical lifetime of the plant 5 Under this assumption, the fraction of the value remaining at the end of the time horizon can be determined as follows:

$$
S_{g, p}^{F R A C}=\max \left[\frac{\left(1-(1+r)^{\left(S Y_{p}+T_{g}^{L I F E}-E O H-1\right)}\right)}{\left(1-(1+r)^{T_{g}^{L I F E}}\right)}, 0\right]
$$

Here, $T_{g}^{L I F E}$ represents the technical lifetime of a certain technology, whereas EOH represents the final year in the considered time horizon.

The equivalent lump-sum fixed operations and maintenance costs related to investments made in period $p$ are also directly related to the investment decisions 6

$$
\begin{aligned}
c_{p}^{f o m} & =\sum_{g \in \mathcal{G}} \sum_{y=S Y_{p}}^{\min \left(S Y_{p}+T_{g}^{L I F E}-1, E O H\right)}\left(\operatorname{cap}_{g, p}^{n e w} C_{g, p}^{F O M} \frac{1}{(1+r)^{\left(y-M Y_{p}\right)}}\right) \\
& +\sum_{s \in \mathcal{S}} \sum_{y=S Y_{p}}^{\min \left(S Y_{p}+T_{s}^{L I F E}-1, E O H\right)}\left(\operatorname{cap}_{s, p}^{n e w} C_{s, p}^{F O M} \frac{1}{(1+r)^{\left(y-M Y_{p}\right)}}\right) .
\end{aligned}
$$

\subsubsection{Operational costs}

All other terms together form the operational costs. The annual operational cost is approximated via a number of representative intervals $i \in \mathcal{I}$ (e.g., weeks or days). Each representative interval $i$ is assumed to be repeated a number of times $W_{i}$ within a typical year. The fuel and emission related generation costs follow from a linearized

\footnotetext{
${ }^{5}$ As discussed in 38 , the value of specific plants can vary strongly over the lifetime and tends to decrease over time. The assumption taken here to distribute the value homogenously over the lifetime of the plant is thus rather optimistic.

${ }^{6}$ The option to mothball or decommission a plant before the end of its technical lifetime is not considered in the current version of the model.
} 
cost curve for dispatchable generation technology-types $g d \in \mathcal{G} \mathcal{D}$, which accounts for part-load efficiency losses:

$$
\begin{aligned}
c_{p}^{g e n}= & \sum_{y=S Y_{p}}^{E Y_{p}} \frac{1}{(1+r)^{\left(y-M Y_{p}\right)}} \sum_{i \in \mathcal{I}} W_{i} \sum_{t \in \mathcal{T}}\left[\Delta_{t}\right. \\
& \left.\sum_{g d \in \mathcal{G} \mathcal{D}} \sum_{v \in \mathcal{V}}\left(n_{g d, v, p, i, t}^{o n} N C_{g d, v, p}+g_{g d, v, p, i, t} M C_{g d, v, p}\right)\right] .
\end{aligned}
$$

In contrast, the VOM costs are assumed to be directly proportional to the generated electrical energy:

$$
\begin{aligned}
c_{p}^{v o m}= & \sum_{y=S Y_{p}}^{E Y_{p}} \frac{1}{(1+r)^{\left(y-M Y_{p}\right)}} \sum_{i \in \mathcal{I}} W_{i} \sum_{t \in \mathcal{T}}\left[\Delta_{t}\right. \\
& \left.\sum_{g \in \mathcal{G}} \sum_{v \in \mathcal{V}}\left(\operatorname{gen}_{g, v, p, i, t} C_{g, v}^{V O M}+p_{s, v, p, i, t}^{c} C_{s, v}^{V O M}\right)\right] .
\end{aligned}
$$

The start-up and ramping costs respectively follow from:

$$
\begin{aligned}
c_{p}^{s u}= & \sum_{y=S Y_{p}}^{E Y_{p}} \frac{1}{(1+r)^{\left(y-M Y_{p}\right)}} \sum_{i \in \mathcal{I}} W_{i} \sum_{t \in \mathcal{T}}\left[\Delta_{t}\right. \\
& \left.\sum_{g d \in \mathcal{G} \mathcal{D}} \sum_{v \in \mathcal{V}}\left(n_{g d, v, p, i, t}^{s u} C_{g d, v}^{S U}\right)\right], \\
c_{p}^{r a m p}= & \sum_{y=S Y_{p}}^{E Y_{p}} \frac{1}{(1+r)^{\left(y-M Y_{p}\right)}} \sum_{i \in \mathcal{I}} W_{i} \sum_{t \in \mathcal{T}}\left[\Delta_{t}\right. \\
& \left.\sum_{g d \in \mathcal{G} \mathcal{D}} \sum_{v \in \mathcal{V}}\left(\operatorname{ramp}_{g d, v, p, i, t} C_{g d, v}^{R A M P}\right)\right] .
\end{aligned}
$$

The model allows to shed load or not provide the required operating reserves. However, there is a cost assigned to load curtailment or not meeting the required reserve requirements:

$$
\begin{gathered}
c_{p}^{l l}=\sum_{y=S Y_{p}}^{E Y_{p}} \frac{1}{(1+r)^{\left(y-M Y_{p}\right)}} \sum_{i \in \mathcal{I}} W_{i} \sum_{t \in \mathcal{T}}\left[\Delta_{t} l l_{p, i, t} V O L L\right], \\
c_{p}^{l r}=\sum_{y=S Y_{p}}^{E Y_{p}} \frac{1}{(1+r)^{\left(y-M Y_{p}\right)}} \sum_{i \in \mathcal{I}} W_{i} \sum_{t \in \mathcal{T}}\left[\Delta_{t} \sum_{r \in \mathcal{R}}\left(l r_{r, p, i, t} \text { VOLR }\right)\right] .
\end{gathered}
$$


Finally, the support for electrical energy generated by renewable generators $g r \in \mathcal{G R}$ is determined as follows:

$$
\begin{aligned}
v_{p}^{r e s}= & \sum_{y=S Y_{p}}^{E Y_{p}} \frac{1}{(1+r)^{\left(y-M Y_{p}\right)}} \sum_{i \in \mathcal{I}} W_{i} \sum_{t \in \mathcal{T}}\left[\Delta_{t}\right. \\
& \left.\sum_{g r \in \mathcal{G} \mathcal{R}} \sum_{v \in \mathcal{V}}\left(\operatorname{gen}_{g r, v, p, i, t} S_{g, p}\right)\right] .
\end{aligned}
$$

\subsection{System constraints}

A number of system constraints need to be fulfilled. First and foremost, supply and demand of electricity must be in balance at all times:

$\sum_{g \in \mathcal{G}} \sum_{v \in \mathcal{V}} g e n_{g, v, p, i, t}+\sum_{s \in \mathcal{S}} \sum_{v \in \mathcal{V}} p_{s, v, p, i, t}^{d}+l l_{p, i, t}=D_{p, i, t}+\sum_{s \in \mathcal{S}} \sum_{v \in \mathcal{V}} p_{s, v, p, i, t}^{c} \quad \forall p \in \mathcal{P}, i \in \mathcal{I}, t \in \mathcal{T}$.

In addition, a planning reserve margin is introduced to ensure generation adequacy. In the current model, only thermal power plants contribute to the planning reserve margin:

$$
\sum_{g d \in \mathcal{G} \mathcal{D}} \sum_{v \in \mathcal{V}} c a p_{g d, v, p}^{a v} \geq \bar{D}_{p}(1+P M) \quad \forall p \in \mathcal{P} .
$$

To deal with contingencies and forecast errors in demand and supply, different types of operating reserves need to be procured. In the standard version of the model, the operating reserves are assumed to be proportional to the demand and scheduled intermittent renewable electricity generation (REF case). Only upward reserves are considered. In addition, only thermal generators and storage technology-types are allowed to provide upward reserves.

$$
\begin{aligned}
& \sum_{g d \in \mathcal{G} \mathcal{D}} \sum_{v \in \mathcal{V}} r_{r, g d, v, p, i, t}^{+}+\sum_{s \in \mathcal{S}} \sum_{v \in \mathcal{V}} r_{r, s, v, p, i, t}^{+}+l r_{r, p, i, t} \\
& \geq R_{r}^{D E M} D_{p, i, t}+\sum_{g r i \in \mathcal{G} \mathcal{R} \mathcal{I}} \sum_{v \in \mathcal{V}}\left(R_{r, g r i}^{F E} g^{2 E} n_{g r i, v, p, i, t}\right) \quad \forall r \in \mathcal{R}, p \in \mathcal{P}, i \in \mathcal{I}, t \in \mathcal{T} .
\end{aligned}
$$

For each reserve category, a certain fraction might need to be provided by spinning units:

$$
\begin{aligned}
& \sum_{g d \in \mathcal{G} \mathcal{D}} \sum_{v \in \mathcal{V}} r_{r, g d, v, p, i, t}^{+, s p i n}+\sum_{s s \in \mathcal{S} \mathcal{S}} \sum_{v \in \mathcal{V}} r_{r, s s, v, p, i, t}^{+}+\sum_{s m \in \mathcal{S} \mathcal{M}} \sum_{v \in \mathcal{V}}\left(r_{r, s m, v, p, i, t}^{+, s p i n, c}+r_{r, s m, v, p, i, t}^{+, s p i n, d}\right) \\
& \geq S_{r}^{S P I N}\left(R_{r}^{D E M} D_{p, i, t}+\sum_{g r i \in \mathcal{G} \mathcal{R} \mathcal{I}} \sum_{v \in \mathcal{V}}\left(R_{r, g r i}^{F E} g e n_{g r i, v, p, i, t}\right)-l r_{r, p, i, t}\right) \\
& \forall r \in \mathcal{R}, p \in \mathcal{P}, i \in \mathcal{I}, t \in \mathcal{T} .
\end{aligned}
$$

For storage technology-types, it is assumed that all reserves provided by battery energy storage systems (BAT) $s s \in \mathcal{S S}$ are sufficiently fast for the provision of spinning reserves. In contrast, pumped-hydro storage (PHS) plants can only provide spinning reserves while charging or discharging.

In an extended version of the model, which is used in the so-called 'Curt' sensitivity case (see Tab. 8), intermittent renewable generators are in certain circumstances allowed 
to provide upward reserves. Additionally, in this version of the model, the sizing of forecast errors is adapted to better account for the effective exposure to forecast errors whenever there is curtailment. The mathematical formulation is described in Section 3.5.

\subsection{Technological constraints}

\subsubsection{Thermal power plants}

Maintenance and life time.

The power generation of thermal power plants is constrained by the installed capacity and technical constraints. First of all, scheduled as well as unplanned outages are taken into account in a stylized fashion by derating the installed capacity with an availability factor $A F_{g d / s, v}$. Additionally, due to the fact that certain periods span multiple years, it can be the case that a certain plant reaches the end of its assumed lifetime somewhere within this period. In this case, it is assumed that a fraction $C P T_{g / s, v, p}$ of the capacity installed in period $v$ is available throughout period $p$ :

$$
c a p_{g d, v, p}^{a v} \leq C P T_{g d, v, p} A F_{g d, v} c a p_{g d, v}^{n e w} \quad \forall g d \in \mathcal{G D}, v \in \mathcal{V}, p \in \mathcal{P} .
$$

The available number of units is directly proportional to the available capacity:

$$
n_{g d, v, p}^{a v} \leq \frac{\operatorname{cap}_{g d, v, p}^{a v}}{\bar{P}_{g d, v}} \quad \forall g d \in \mathcal{G} \mathcal{D}, v \in \mathcal{V}, p \in \mathcal{P} .
$$

Finally, the number of online units, together with those procured to provide non-spinning reserves are restricted by the available units:

$$
n_{g d, v, p, i, t}^{o n}+n_{g d, v, p, i, t}^{+, n s} \leq n_{g d, v, p}^{a v} \quad \forall g d \in \mathcal{G D}, v \in \mathcal{V}, p \in \mathcal{P}, i \in \mathcal{I}, t \in \mathcal{T} .
$$

Logical commitment conditions.

The number of online/spinning units can be changed by starting up or shutting down a number of units:

$$
n_{g d, v, p, i, t+1}^{o n}=n_{g d, v, p, i, t}^{o n}+n_{g d, v, p, i, t}^{s u}-n_{g d, v, p, i, t}^{s d} \quad \forall g d \in \mathcal{G} \mathcal{D}, v \in \mathcal{V}, p \in \mathcal{P}, i \in \mathcal{I}, t \in \mathcal{T} .
$$

Finally, the discrete nature of power plants is reflected by restricting the variables representing a number of units to natural numbers, i.e., integer variables are used in the MIP model version:

$n_{g d, v, p}^{a v}, n_{g d, v, p, i, t}^{o n}, n_{g d, v, p, i, t}^{s u}, n_{g d, v, p, i, t}^{s d}, n_{g d, v, p, i, t}^{+, n s} \in \mathbb{Z}_{0}^{+} \quad \forall g d \in \mathcal{G D}, v \in \mathcal{V}, p \in \mathcal{P}, i \in \mathcal{I}, t \in \mathcal{T}$.

In the LP version of the model, the integral nature of these variables is relaxed, i.e., continuous variables are used.

Generation level constraints.

The power plant output is defined as:

$$
g e n_{g d, v, p, i, t}=n_{g d, v, p, i, t}^{o n} \underline{P}_{g d, v}+g_{g d, v, p, i, t} \quad \forall g d \in \mathcal{G D}, v \in \mathcal{V}, p \in \mathcal{P}, i \in \mathcal{I}, t \in \mathcal{T} .
$$


This constraint also ensures that whenever a plant is online/spinning, it must be operated above a certain minimum power output (i.e., the minimum stable operating point). In addition, the maximum power output is restricted by the rated power:

$$
\begin{aligned}
& \operatorname{gen}_{g d, v, p, i, t}+\sum_{r \in \mathcal{R}} r_{r, g d, v, p, i, t}^{+, s p i n} \leq\left(n_{g d, v, p, i, t}^{o n}-n_{g d, v, p, i, t-1}^{s u}-n_{g d, v, p, i, t}^{s d}\right) \bar{P}_{g d, v} \\
& +n_{g d, v, p, i, t-1}^{s u} S U_{g d, v}+n_{g d, v, p, i, t}^{s d} S D_{g d, v} \quad \forall g d \in \mathcal{G} \mathcal{D}, v \in \mathcal{V}, p \in \mathcal{P}, i \in \mathcal{I}, t \in \mathcal{T} .
\end{aligned}
$$

In the above constraint, the maximum power output of units which have been online in the previous time step and remain to be online in the following time step is restricted by the rated power output, whereas directly after a start-up and before a shut-down, the maximum power is constrained to $S U_{g d, v}$ and $S D_{g d, v}$ respectively. This constraint also makes sure that whenever spinning upward reserves are procured, sufficient head room is available.

\section{Ramping constraints.}

Changes in generation level are constrained by ramping limits. In a CUC formulation, the power output can be adapted by changing the power output of spinning units, starting up additional units and shutting down units simultaneously. Therefore, the ramping constraint needs to be adjusted correspondingly. In addition, the possible activation of spinning reserves affects the ramp, and is therefore taken into account. The upward and downward ramping constraints respectively become:

$$
\begin{aligned}
& \operatorname{gen}_{g d, v, p, i, t+1}-g e n_{g d, v, p, i, t}+\sum_{r \in \mathcal{R}} r_{r, g d, v, p, i, t+1}^{+, s p i n} \leq \\
& \left(n_{g d, v, p, i, t}^{o n}-n_{g d, v, p, i, t}^{s d}\right) \bar{P}_{g d, v} \frac{R_{g d, v}}{100} \Delta_{t} \\
& -n_{g d, v, p, i, t}^{s d} \underline{P}_{g d, v}+n_{g d, v, p, i, t}^{s u} S U_{g d, v} \quad \forall g d \in \mathcal{G} \mathcal{D}, v \in \mathcal{V}, p \in \mathcal{P}, i \in \mathcal{I}, t \in \mathcal{T}, \\
& g e n_{g d, v, p, i, t}-g e n_{g d, v, p, i, t+1}+\sum_{r \in \mathcal{R}} r_{r, g d, v, p, i, t}^{+, s p i n} \leq \\
& \left(n_{g d, v, p, i, t}^{o n}-n_{g d, v, p, i, t}^{s d}\right) \bar{P}_{g d, v} \frac{R_{g d, v}}{100} \Delta_{t} \quad \\
& -n_{g d, v, p, i, t}^{s u} \underline{P}_{g d, v}+n_{g d, v, p, i, t}^{s d} S D_{g d, v} \quad \forall g d \in \mathcal{G D}, v \in \mathcal{V}, p \in \mathcal{P}, i \in \mathcal{I}, t \in \mathcal{T},
\end{aligned}
$$

Due to the fact that in a CUC formulation, the power ramps do not directly follow from the changes in total power output, the ramps need to be determined for assigning ramping costs:

$$
\begin{aligned}
& \operatorname{ramp}_{g d, v, p, i, t} \geq \operatorname{gen}_{g d, v, p, i, t+1}-\text { gen }_{g d, v, p, i, t} \\
& +n_{g d, v, p, i, t}^{s d} \underline{P}_{g d, v}-n_{g d, v, p, i, t}^{s u} S U_{g d, v} \quad \forall g d \in \mathcal{G} \mathcal{D}, v \in \mathcal{V}, p \in \mathcal{P}, i \in \mathcal{I}, t \in \mathcal{T}, \\
& r a m p_{g d, v, p, i, t} \geq g e n_{g d, v, p, i, t}-g e n_{g d, v, p, i, t+1} \\
& +n_{g d, v, p, i, t}^{s u} \underline{P}_{g d, v}-n_{g d, v, p, i, t}^{s d} S D_{g d, v} \quad \forall g d \in \mathcal{G D}, v \in \mathcal{V}, p \in \mathcal{P}, i \in \mathcal{I}, t \in \mathcal{T}
\end{aligned}
$$


Minimum up and down time constraints.

Minimum up and down time constraints respectively force units starting up/shutting down to remain online/offline for a minimum amount of time. These constraints are formulated as follows:

$$
\begin{aligned}
n_{g d, v, p, i, t}^{s d} \leq & n_{g d, v, p, i, t}^{o n}-\sum_{t^{\prime}=1}^{M U T-1} n_{g d, v, p, i, t-t^{\prime}}^{s u} \quad \forall g d \in \mathcal{G D}, v \in \mathcal{V}, p \in \mathcal{P}, i \in \mathcal{I}, t \in \mathcal{T}, \\
& n_{g d, v, p, i, t}^{s u}+\sum_{r \in \mathcal{R}} n_{g d, v, p, i, t}^{+, n s} \leq n_{g d, v, p}^{a v}-n_{g d, v, p, i, t}^{o n} \\
& -\sum_{t^{\prime}=1}^{M D T-1} n_{g d, v, p, i, t-t^{\prime}}^{s d} \quad \forall g d \in \mathcal{G} \mathcal{D}, v \in \mathcal{V}, p \in \mathcal{P}, i \in \mathcal{I}, t \in \mathcal{T} .
\end{aligned}
$$

Eq. 29 can be read as the number of units shutting down is restricted to the online units which were not recently started up. Similarly, Eq. (30) can be read as the number of units starting up is restricted to the available offline units which were not recently shut down.

Provision of reserves.

The upward reserves provided by thermal generators consist of both spinning and nonspinning reserves:

$$
r_{r, g d, v, p, i, t}^{+}=r_{r, g d, v, p, i, t}^{+, s p i n}+r_{r, g d, v, p, i, t}^{+, n s} \quad \forall r \in \mathcal{R}, g d \in \mathcal{G D}, v \in \mathcal{V}, p \in \mathcal{P}, i \in \mathcal{I}, t \in \mathcal{T}
$$

The provision of spinning reserves is constrained by the available head room on the one hand (see Eq. (24)), and the ability to ramp within the required time for activation. A single generator can provide multiple types of reserve requirements in a given time step. In this case, the ramping capability of this generator must be sufficient to cover the ramps required for the provision of the different types of reserves. For simplicity, we assume that the ramping constraint for a given type of reserves is not significantly impacted by possible ramps that need to be realized for slower types of reserves. This implies that for the fastest reserve type, only the reserves procured for this reserve type need to be considered for the ramping constraint. For the other reserve types, the reserves procured for faster reserve types are also taken into consideration.

$$
\begin{aligned}
& \sum_{r \in \mathcal{R}: T_{r}^{A C T} \leq T_{r^{\prime}}^{A C T}} r_{r, g d, v, p, i, t}^{+, s p i n} \leq\left(n_{g d, v, p, i, t}^{o n}-n_{g d, v, p, i, t}^{s d}\right) \bar{P}_{g d, v} \frac{R_{g d, v}}{100} T_{r^{\prime}}^{A C T} \\
& \forall r^{\prime} \in \mathcal{R}, g d \in \mathcal{G D}, v \in \mathcal{V}, p \in \mathcal{P}, i \in \mathcal{I}, t \in \mathcal{T} .
\end{aligned}
$$

Finally, the provision of non-spinning (fast-starting) reserves is constrained to those technology-types for which the start-up time is below the required activation time:

$$
\sum_{r \in \mathcal{R}: T_{r}^{A C T}<S U T_{g d, v}} r_{r, g d, v, p, i, t}^{+, n s}=0 \quad \forall g d \in \mathcal{G D}, v \in \mathcal{V}, p \in \mathcal{P}, i \in \mathcal{I}, t \in \mathcal{T} .
$$

For units starting up sufficiently fast, the reserves are constrained by the rated power:

$$
\sum_{r \in \mathcal{R}: T_{r}^{A C T} \geq S U T_{g d, v}} r_{r, g d, v, p, i, t}^{+, n s} \leq n_{g d, v, p, i, t}^{+, n s} \bar{P}_{g d, v} \quad \forall g d \in \mathcal{G D}, v \in \mathcal{V}, p \in \mathcal{P}, i \in \mathcal{I}, t \in \mathcal{T} .
$$




\subsubsection{Intermittent renewable energy sources}

For IRES, the power generation is constrained by the availability of the resource (i.e., wind or solar irradiation). Curtailment is allowed whenever necessary or cost-effective:

gen $_{\text {gri }, v, p, i, t}+$ curt $_{\text {gri }, v, p, i, t}=\operatorname{cap}_{\text {gri }, v}^{\text {new }} C P T_{\text {gri }, v, p} C F_{\text {gri } i, p, p, i, t} \quad \forall g r i \in \mathcal{G R}, v \in \mathcal{V}, p \in \mathcal{P}, i \in \mathcal{I}, t \in \mathcal{T}$.

\subsubsection{Storage technologies}

For storage technology-types, separate investments can be made for power capacity (charging and discharging facilities) and energy capacity. However, for each storage technology, the ratio between power and energy capacity is bound to certain limits, specified via a minimal and a maximal discharge duration:

$$
\frac{c a p_{s, v}^{n e w}}{\eta_{s, v}} \overline{D U R}_{s, v} \geq c a p_{s, v}^{n e w, e} \geq \frac{c a p_{s, v}^{n e w}}{\eta_{s, v}} \underline{D U R}_{s, v}
$$

The charging and discharging capacity is assumed to be identical.

Since batteries do not have a minimum operating point and individual units are small, no commitment variables are used to model batteries. In contrast, for PHS technologytypes, integer commitment variables are used in the MIP model version (see Eq. (44)), and continuous variables in the LP model version.

\section{Maintenance.}

Again, scheduled as well as unplanned outages are taken into account in a stylized fashion by derating the installed capacity with an availability factor $A F_{s}$ :

$$
\operatorname{cap}_{s, v, p}^{a v} \leq C P T_{s, v, p} A F_{s, v} \operatorname{cap}_{s, v}^{\text {new }} \quad \forall s \in \mathcal{S}, v \in \mathcal{V}, p \in \mathcal{P}
$$

For PHS, the available number of charging/discharging units is directly proportional to the available capacity:

$$
\begin{aligned}
& n_{s m, v, p}^{c, a v} \leq \frac{c a p_{s m, v, p}^{a v}}{\bar{P}_{s m, v}} \quad \forall s m \in \mathcal{S M}, v \in \mathcal{V}, p \in \mathcal{P}, \\
& n_{s m, v, p}^{d, a v} \leq \frac{c a p_{s m, v, p}^{a v}}{\bar{P}_{s m, v}} \quad \forall s m \in \mathcal{S M}, v \in \mathcal{V}, p \in \mathcal{P} .
\end{aligned}
$$

The available units in turn restrict the number of online charging/discharging units:

$$
\begin{gathered}
n_{s m, v, p, i, t}^{c, o n} \leq n_{s m, v, p}^{c, a v} \quad \forall s m \in \mathcal{S M}, v \in \mathcal{V}, p \in \mathcal{P}, i \in \mathcal{I}, t \in \mathcal{T}, \\
n_{s m, v, p, i, t}^{d, o n}+n_{s m, v, p, i, t}^{+, s u, d} \leq n_{s m, v, p}^{d, a v} \quad \forall s m \in \mathcal{S} \mathcal{M}, v \in \mathcal{V}, p \in \mathcal{P}, i \in \mathcal{I}, t \in \mathcal{T} .
\end{gathered}
$$




\section{Logical conditions.}

The number of online charging or discharging PHS units can be changed by starting up or shutting down a number of units:

$n_{s m, v, p, i, t+1}^{c, o n}=n_{s m, v, p, i, t}^{c, o n}+n_{s m, v, p, i, t}^{c, s u}-n_{s m, v, p, i, t}^{c, s d} \quad \forall s m \in \mathcal{S} \mathcal{M}, v \in \mathcal{V}, p \in \mathcal{P}, i \in \mathcal{I}, t \in \mathcal{T}$,

$n_{s m, v, p, i, t+1}^{d, o n}=n_{s m, v, p, i, t}^{d, o n}+n_{s m, v, p, i, t}^{d, s u}-n_{s m, v, p, i, t}^{d, s d} \quad \forall s m \in \mathcal{S M}, v \in \mathcal{V}, p \in \mathcal{P}, i \in \mathcal{I}, t \in \mathcal{T}$.

In addition, the discrete nature of the pumps and turbines is reflected by restricting the variables representing a number of units to natural numbers, i.e., integer variables are used in the MIP model version:

$$
\begin{aligned}
& n_{s m, v, p}^{c, a v}, n_{s m, v, p, i, t}^{c, o n}, n_{s m, v, p, i, t}^{c, s u}, n_{s m, v, p, i, t}^{c, s d}, n_{s m, v, p}^{d, a v}, n_{s m, v, p, i, t}^{d, o n}, n_{s m, v, p, i, t}^{d, s u}, \\
& n_{s m, v, p, i, t}^{d, s d}, n_{s m, v, p, i, t}^{+, s u, d}, n_{s m, v, p, i, t}^{+, s d, c} \in \mathbb{Z}_{0}^{+} \quad \forall s m \in \mathcal{S M}, v \in \mathcal{V}, p \in \mathcal{P}, i \in \mathcal{I}, t \in \mathcal{T} .
\end{aligned}
$$

Again, the integrality conditions are relaxed in the LP model version, i.e., continuous variables are used.

\section{Energy balance and reservoir.}

Storage technology-types can transfer energy on both the short and the longer term. Since the temporal representation of the model is based on using a limited number representative intervals, special attention is needed to consider arbitrage opportunities over longer intervals (e.g., months or seasons). To allow arbitraging over longer time frames, energy transfer between different representative intervals is required. This implies that within each representative interval, the model must allow a net change in the energy content in the reservoir, such that for instance the storage is allowed to be charged during the first representative interval and gradually discharged during the second and third representative intervals. Recall further that each representative interval is assumed to occur a number of times within a single year (represented by parameter $\left.W_{i}\right)^{7}$. However, assumptions need to be made regarding how the occurrence of a certain representative interval is spread over the course of the year. In the current model version, it is assumed that each representative interval $i$ is repeated a number of times equal to its weight $W_{i}$, before the subsequent representative interval $i+1$ starts (which in turn is repeated a number of times $\left.W_{i+1}\right)$.

Under these assumptions, the relationship between the energy content at the start of each representative interval and the charging and discharging decisions within the corresponding representative intervals is expressed as follows:

$e_{s, v, p, i+1, t=1}^{f}=e_{s, v, p, i, t=1}^{f}+\sum_{t \in \mathcal{T}}\left(W_{i} \Delta_{t}\left(p_{s, v, p, i, t}^{c} \sqrt{\eta_{s, v}}-\frac{p_{s, v, p, i, t}^{d}}{\sqrt{\eta_{s, v}}}\right)\right) \quad \forall s \in \mathcal{S}, v \in \mathcal{V}, p \in \mathcal{P}, i \in \mathcal{I}$.

\footnotetext{
${ }^{7}$ Note further that the charging/discharging pattern is identical within each repetition of a certain representative interval, i.e., there is only a single dispatch variable per representative interval and time step.
} 
To ensure that within each representative interval, the energy content does not exceed its limits, additional constraints are required. Although the charging and discharging pattern is the same for each repetition of the representative interval, due to the option of having a net increase/decrease within each representative interval, the energy content in the reservoir can increase/decrease as the representative interval is repeated. Given that there is either a net increase or a net decrease of the energy content in the reservoir for all repetitions of a single representative interval, it can be derived that if the energy content would violate its limits during certain repetitions of the representative interval, this would definitively be the case within the first and/or last repetition of the interval. Assume for instance that there is a net increase in the energy content over the course of the representative interval. In this case, if the upper limit of the energy reservoir would be exceeded, this would definitively be exceeded in the last repetition of the representative interval. In addition, if the lower limit would be violated, this would definitively be the case in the first repetition of the representative interval. This is visualized in Fig. 3 . A similar reasoning can be followed when a net decrease in the energy content is assumed.

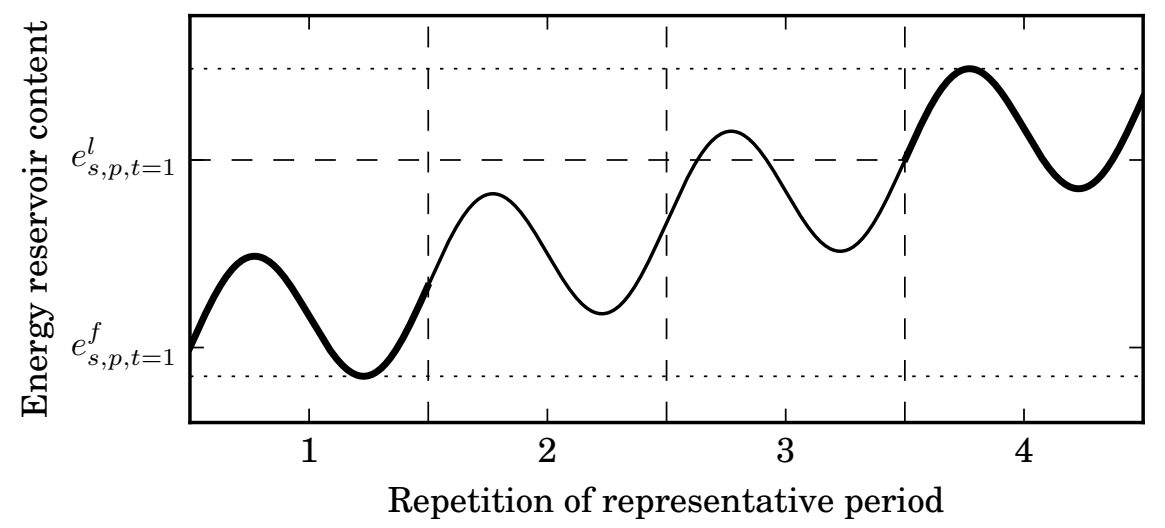

Figure 3: Methodology for modeling storage arbitrage opportunities within the representative interval and across different representative intervals. In this example, the storage performs some arbitrage within the representative interval, but there is also a net increase in the stored energy over the course of the representative interval, which can be utilized in subsequent intervals, i.e., there is the possibility to arbitrage between representative intervals. To ensure that the energy content limits are not exceeded, it is sufficient to guarantee that in the first and the last repetition of the representative interval the limits are not exceeded. This is visualized by the dotted lines which indicate the minimum and maximum stored energy level.

Thus to make sure that the energy content does not violate its limits, the energy content within the first and the last repetition of the representative interval must first be determined:

$e_{s, v, p, i, t+1}^{f}=e_{s, v, p, i, t}^{f}+\Delta_{t}\left(p_{s, v, p, i, t}^{c} \sqrt{\eta_{s, v}}-\frac{p_{s, v, p, i, t}^{d}}{\sqrt{\eta_{s, v}}}\right) \quad \forall s \in \mathcal{S}, v \in \mathcal{V}, p \in \mathcal{P}, i \in \mathcal{I}, t \in \mathcal{T}: t \neq\|\mathcal{T}\|$, 
$e_{s, v, p, i, t=1}^{l}=e_{s, v, p, i, t=1}^{f}+\sum_{t \in \mathcal{T}}\left(\left(W_{i}-1\right) \Delta_{t}\left(p_{s, v, p, i, t}^{c} \sqrt{\eta_{s, v}}-\frac{p_{s, v, p, i, t}^{d}}{\sqrt{\eta_{s, v}}}\right)\right) \quad \forall s \in \mathcal{S}, v \in \mathcal{V}, p \in \mathcal{P}, i \in \mathcal{I}$,

$e_{s, i, t+1}^{l}=e_{s, v, p, i, t}^{l}+\Delta_{t}\left(p_{s, v, p, i, t}^{c} \sqrt{\eta_{s, v}}-\frac{p_{s, v, p, i, t}^{d}}{\sqrt{\eta_{s, v}}}\right) \quad \forall s \in \mathcal{S}, v \in \mathcal{V}, p \in \mathcal{P}, i \in \mathcal{I}, t \in \mathcal{T}: t \neq\|\mathcal{T}\|$

Next, the minimum and maximum energy content limits can be enforced for the first and last repetition of each representative interval:

$$
\begin{aligned}
& c a p_{s, v}^{\text {new }, e}-\Delta_{t} p_{s, v, p, i, t}^{c} \sqrt{\eta_{s, v}} \geq e_{s, v, p, i, t}^{f} \geq \\
& \left.\Delta_{t} \frac{p_{s, v, p, i, t}^{d}}{\sqrt{\eta_{s, v}}}\right)+\sum_{r \in \mathcal{R}}\left(r_{r, s, v, p, i, t}^{+, d} T_{r}^{D U R}\right) \quad \forall s \in \mathcal{S}, v \in \mathcal{V}, p \in \mathcal{P}, i \in \mathcal{I}, t \in \mathcal{T}, \\
& \operatorname{cap}_{s, v}^{\text {new,e }}-\Delta_{t} p_{s, v, p, i, t}^{c} \sqrt{\eta_{s, v}} \geq e_{s, v, p, i, t}^{l} \geq \\
& \left.\Delta_{t} \frac{p_{s, v, p, i, t}^{d}}{\sqrt{\eta_{s, v}}}\right)+\sum_{r \in \mathcal{R}}\left(r_{r, s, v, p, i, t}^{+, d} T_{r}^{D U R}\right) \quad \forall s \in \mathcal{S}, v \in \mathcal{V}, p \in \mathcal{P}, i \in \mathcal{I}, t \in \mathcal{T} .
\end{aligned}
$$

Eq. (49)-(50) also make sure that whenever spinning reserves are procured from storage systems, the energy content limits would not be violated if the procured reserves would need to be activated for a duration $T_{r}^{D U R}$.

It must be noted that whenever each representative interval is repeated rather frequently (for instance if a low number of representative intervals is used and/or the duration of each representative interval is short), the assumption of having all repetitions of each representative interval directly after each other will likely overly restrict the ability to arbitrage over longer time frames. This because, due to the high number of repetitions, a small net increase/decrease of the energy content over the course of a single repetition of a representative interval can already lead to a high net increase/decrease of the energy content over all repetitions of this representative interval. As a consequence, the energy reservoir limits can quickly become binding, thereby restricting the net increase/decrease within each representative interval to small amounts. Further research is required to analyze and improve the modeling of longer term storage when using representative intervals.

Charging/discharging level constraints.

For batteries, the charging/discharging level is constrained by the available capacity and the procured upward reserves. Note that the upward reserves that can be provided while charging correspond to a decrease of the charging power.

$$
p_{s s, v, p, i, t}^{c} \leq c a p_{s s, v, p}^{a v} \quad \forall s s \in \mathcal{S} \mathcal{S}, v \in \mathcal{V}, p \in \mathcal{P}, i \in \mathcal{I}, t \in \mathcal{T},
$$




$$
\begin{gathered}
p_{s s, v, p, i, t}^{d}+\sum_{r \in \mathcal{R}} r_{r, s s, v, p, i, t}^{+, d} \leq c a p_{s s, v, p}^{a v} \quad \forall s s \in \mathcal{S} \mathcal{S}, v \in \mathcal{V}, p \in \mathcal{P}, i \in \mathcal{I}, t \in \mathcal{T} . \\
p_{s s, v, p, i, t}^{c} \geq \sum_{r \in \mathcal{R}} r_{s s, v, p, i, t}^{+, c} \quad \forall s s \in \mathcal{S} \mathcal{S}, v \in \mathcal{V}, p \in \mathcal{P}, i \in \mathcal{I}, t \in \mathcal{T} .
\end{gathered}
$$

For PHS, the maximum charging/discharging power is dependent on the number of online units:

$$
\begin{gathered}
p_{s m, v, p, i, t}^{c} \leq n_{s m, v, p, i, t}^{c, o n} \bar{P}_{s m, v} \quad \forall s m \in \mathcal{S} \mathcal{M}, v \in \mathcal{V}, p \in \mathcal{P}, i \in \mathcal{I}, t \in \mathcal{T}, \\
p_{s m, v, p, i, t}^{d}+\sum_{r \in \mathcal{R}} r_{r, s m, v, p, i, t}^{+, s p i n, d} \leq n_{s m, v, p, i, t}^{d, o n} \bar{P}_{s m, v} \quad \forall s m \in \mathcal{S} \mathcal{M}, v \in \mathcal{V}, p \in \mathcal{P}, i \in \mathcal{I}, t \in \mathcal{T} .
\end{gathered}
$$

Similar to the thermal power plants, both in pumping and turbining mode, minimum stable operating point restrictions must be respected:

$$
\begin{gathered}
p_{s m, v, p, i, t}^{c} \geq n_{s m, v, p, i, t}^{c, o n} \underline{P}_{s m, v}^{C}+\sum_{r \in \mathcal{R}} r_{r, s m, v, p, i, t}^{+, s p i n, c} \quad \forall s m \in \mathcal{S} \mathcal{M}, v \in \mathcal{V}, p \in \mathcal{P}, i \in \mathcal{I}, t \in \mathcal{T}, \\
p_{s m, v, p, i, t}^{d} \geq n_{s m, v, p, i, t}^{d, o n} \underline{P}_{s m, v}^{D} \quad \forall s m \in \mathcal{S} \mathcal{M}, v \in \mathcal{V}, p \in \mathcal{P}, i \in \mathcal{I}, t \in \mathcal{T},
\end{gathered}
$$

Ramping constraints.

For batteries, no ramping constraints are considered. In contrast, PHS face upward and downward ramping constraints, both while charging and while discharging:

$$
\begin{aligned}
& p_{s m, v, p, i, t+1}^{c}-p_{s m, v, p, i, t}^{c}+\sum_{r \in \mathcal{R}} r_{r, s m, v, p, i, t}^{+, s p i n, c} \leq\left(n_{s m, v, p, i, t}^{c, o n}-n_{s m, v, p, i, t}^{c, s d}\right) \bar{P}_{s m, v} \frac{R_{s m, v}}{100} \Delta_{t} \\
& -n_{s m, v, p, i, t}^{c, s d} \underline{P}_{s m, v}^{C}+n_{s m, v, p, i, t}^{c, s u} \bar{P}_{s m, v} \quad \forall s m \in \mathcal{S M}, v \in \mathcal{V}, p \in \mathcal{P}, i \in \mathcal{I}, t \in \mathcal{T}, \\
& p_{s m, v, p, i, t+1}^{d}-p_{s m, v, p, i, t}^{d}+\sum_{r \in \mathcal{R}} r_{s m, v, p, i, t+1}^{+, s p i n, d} \leq\left(n_{s m, v, p, i, t}^{d, o n}-n_{s m, v, p, i, t}^{d, s d}\right) \bar{P}_{s m, v} \frac{R_{s m, v}}{100} \Delta_{t} \\
& -n_{s m, v, p, i, t}^{d, s d} \underline{P}_{s m, v}^{D}+n_{s m, v, p, i, t}^{d, s u} \bar{P}_{s m, v} \quad \forall s m \in \mathcal{S} \mathcal{M}, v \in \mathcal{V}, p \in \mathcal{P}, i \in \mathcal{I}, t \in \mathcal{T}, \\
& p_{s m, v, p, i, t}^{c}-p_{s m, v, p, i, t+1}^{c}+\sum_{r \in \mathcal{R}} r_{s m, v, p, i, t+1}^{+, s p i n, c} \leq\left(n_{s m, v, p, i, t}^{c, o n}-n_{s m, v, p, i, t}^{c, s d}\right) \bar{P}_{s m, v} \frac{R_{s m, v}}{100} \Delta_{t} \\
& +n_{s m, v, p, i, t}^{c, s d} \bar{P}_{s m, v}-n_{s m, v, p, i, t}^{c, s u} \underline{P}_{s m, v}^{C} \quad \forall s m \in \mathcal{S} \mathcal{M}, v \in \mathcal{V}, p \in \mathcal{P}, i \in \mathcal{I}, t \in \mathcal{T}, \\
& p_{s m, v, p, i, t}^{d}-p_{s m, v, p, i, t+1}^{d}+\sum_{r \in \mathcal{R}} r_{r, s m, v, p, i, t}^{+, s p i n, d} \leq\left(n_{s m, v, p, i, t}^{d, o n}-n_{s m, v, p, i, t}^{d, s s}\right) \bar{P}_{s m, v} \frac{R_{s m, v}}{100} \Delta_{t} \\
& +n_{s m, v, p, i, t}^{d, s d} \bar{P}_{s m, v}-n_{s m, v, p, i, t}^{d, s u} \underline{P}_{s m, v}^{D} \quad \forall s m \in \mathcal{S M}, v \in \mathcal{V}, p \in \mathcal{P}, i \in \mathcal{I}, t \in \mathcal{T} .
\end{aligned}
$$


Provision of reserves.

Upward reserves by storage technology-types can be provided by increasing the discharging power or decreasing the scheduled charging power:

$$
r_{r, s, v, p, i, t}^{+}=r_{r, s, v, p, i, t}^{+, c}+r_{r, s, v, p, i, t}^{+, d} \quad \forall r \in \mathcal{R}, s \in \mathcal{S}, v \in \mathcal{V}, p \in \mathcal{P}, i \in \mathcal{I}, t \in \mathcal{T}
$$

The provision of reserves by batteries is restricted by the energy content in the reservoir (Eq. (49)-(50p), the available head room while discharging (Eq. (52) ) and the amount of scheduled charging power (Eq. (53p).

For PHS, the procurement of reserves by increasing the level of discharging is also restricted by the energy content in the reservoir (Eq. 49p-(50). To consider technical constraints for PHS, the provision of upward reserves while charging/discharging is divided into spinning and non-spinning reserves:

$$
\begin{array}{ll}
r_{r, s m, v, p, i, t}^{+, c}=r_{r, s m, v, p, i, t}^{+, s p i n, c}+r_{r, s m, v, p, i, t}^{+, n s, c} & \forall r \in \mathcal{R}, s m \in \mathcal{S} \mathcal{M}, v \in \mathcal{V}, p \in \mathcal{P}, i \in \mathcal{I}, t \in \mathcal{T}, \\
r_{r, s m, v, p, i, t}^{+, d}=r_{r, s m, v, p, i, t}^{+, s p i n, d}+r_{r, s m, v, p, i, t}^{+, n s, d} & \forall r \in \mathcal{R}, s m \in \mathcal{S} \mathcal{M}, v \in \mathcal{V}, p \in \mathcal{P}, i \in \mathcal{I}, t \in \mathcal{T} .
\end{array}
$$

The provision of spinning reserves is restricted by the available head room while discharging (see Eq. (55p), and the minimum stable operating point while charging (see Eq. (56)). In addition, ramping constraints need to be considered (upward ramping while discharging and downward ramping while charging):

$$
\begin{aligned}
& \sum_{r \in \mathcal{R}: T_{r}^{A C T} \leq T_{r^{\prime}}^{A C T}} r_{r, s m, v, p, i, t}^{+, s p i n, d} \leq\left(n_{s m, v, p, i, t}^{d, o n}-n_{s m, v, p, i, t}^{d, s d}\right) \bar{P}_{s m, v} \frac{R_{s m, v}}{100} T_{r^{\prime}}^{A C T} \\
& \forall r^{\prime} \in \mathcal{R}, s m \in \mathcal{S} \mathcal{M}, v \in \mathcal{V}, p \in \mathcal{P}, i \in \mathcal{I}, t \in \mathcal{T} . \\
& \sum_{r \in \mathcal{R}: T_{r}^{A C T} \leq T_{r^{\prime}}^{A C T}} r_{r, s m, v, p, i, t}^{+, s p i n, c} \leq\left(n_{s m, v, p, i, t}^{c, o n}-n_{s m, v, p, i, t}^{c, s d}-n_{s m, v, p, i, t}^{+, s d, c}\right) \\
& \bar{P}_{s m, v} \frac{R_{s m, v}}{100} T_{r^{\prime}}^{A C T} \quad \forall r^{\prime} \in \mathcal{R}, s m \in \mathcal{S} \mathcal{M}, v \in \mathcal{V}, p \in \mathcal{P}, i \in \mathcal{I}, t \in \mathcal{T} .
\end{aligned}
$$

Finally, also the reserves provided by starting up additional discharging units, or shutting down charging units need to be restricted:

$$
\begin{gathered}
\sum_{r \in \mathcal{R}: T_{r}^{A C T}<S U T_{s m, v}^{D}} r_{r, s m, v, p, i, t}^{+, n s, d}=0 \quad \forall s m \in \mathcal{S} \mathcal{M}, v \in \mathcal{V}, p \in \mathcal{P}, i \in \mathcal{I}, t \in \mathcal{T}, \\
\sum_{r \in \mathcal{R}: T_{r}^{A C T}<S D T_{s m, v}^{C}} r_{r, s m, v, p, i, t}^{+, n s, c}=0 \quad \forall s m \in \mathcal{S M}, v \in \mathcal{V}, p \in \mathcal{P}, i \in \mathcal{I}, t \in \mathcal{T}, \\
\sum_{r \in \mathcal{R}: T_{r}^{A C T} \geq S U T_{s m, v}^{D}} r_{r, s m, v, p, i, t}^{+, n s, d} \leq n_{s m, v, p, i, t}^{+, s u, d}, \bar{P}_{s m, v}
\end{gathered}
$$


$\sum_{r \in \mathcal{R}: T_{r}^{A C T} \geq S D T_{s m, v}^{C}} r_{r, s m, v, p, i, t}^{+, n s, c} \leq n_{s m, v, p, i, t}^{+, s d, c} \underline{P}_{s m, v}^{C} \quad \forall s m \in \mathcal{S M}, v \in \mathcal{V}, p \in \mathcal{P}, i \in \mathcal{I}, t \in \mathcal{T}$

Due to the fact that spinning reserves can be provided by reducing the pumping (charging) level down to the minimum stable operating point, non-spinning reserves are restricted to the minimum stable operating point.

\section{5. reserve requirements in the Curt case}

This sections describes the mathematical formulation for the sizing of reserves dependent on the effective exposure to forecast errors and for the provision of upward reserves by IRES. In the remainder of this discussion, we focus on wind. However, the methodology can be directly transferred to other IRES. It must be noted that the presented formulation is highly simplified. It merely serves to get an idea of how results might change whenever the sizing of reserves is directly based on the exposure to forecast errors and IRES are allowed to provide upward reserves to cope with other types of uncertainty.

We make the base assumption that a certain fraction $(1-\alpha)$ of the forecasted wind power $\bar{W}$ can be guaranteed with a reasonable certainty. Following this assumption, the exposure to wind forecast errors is reduced whenever there is more curtailment, as visualized in Fig. 4. In addition, in periods of strong oversupply of wind, the scheduled wind generation might be below the level which can be guaranteed with reasonable certainty. In this case, there is no need to ensure reserves to deal with wind forecast errors. Moreover, the curtailment below the wind generation level which can be guaranteed with reasonable certainty can be used to provide upward reserves to cover other sources of uncertainty. This is visualized in Fig. 5 .

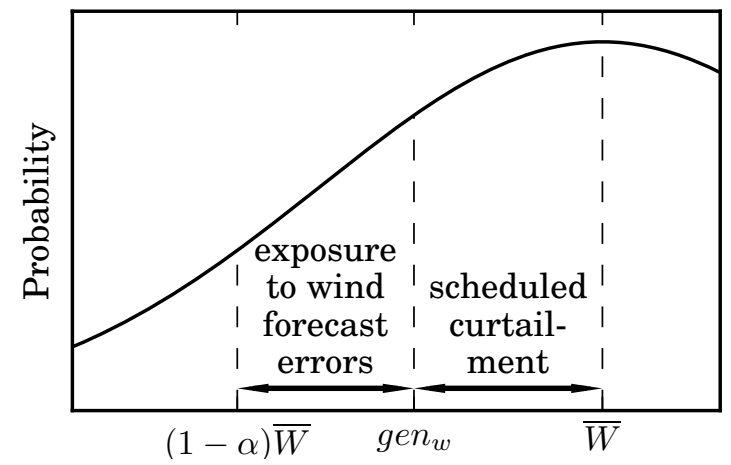

Figure 4: Illustration of the reduction of the need for reserves by scheduled curtailment. A probability distribution of wind generation is presented. The forecasted wind generation when there would be no curtailment is indicated by $\bar{W}$. The wind generation that can be guaranteed with a reasonable certainty is indicated by $(1-\alpha) \bar{W}$, where $\alpha$ represents the uncertain fraction of the forecasted wind generation. The scheduled wind generation is indicated by $g e n_{w}$. 


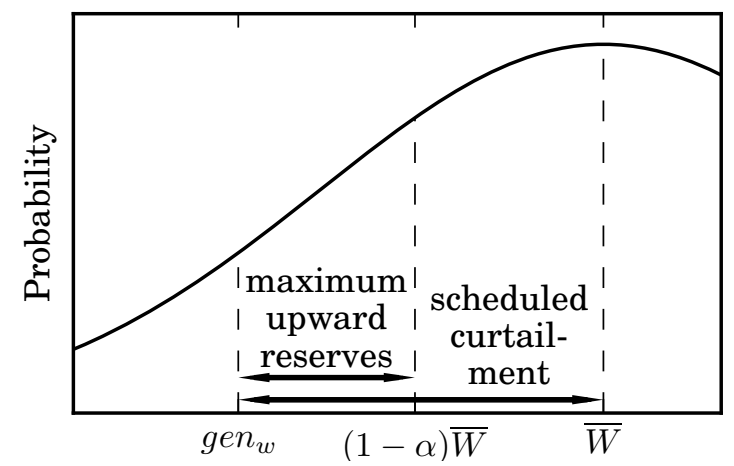

Figure 5: Illustration of the provision of upward reserves by IRES. A probability distribution of wind generation is presented. The forecasted wind generation when there would be no curtailment is indicated by $\bar{W}$. The wind generation that can be guaranteed with a reasonable certainty is indicated by (1 $\alpha) \bar{W}$, where $\alpha$ represents the uncertain fraction of the forecasted wind generation. The scheduled wind generation is indicated by gen $_{w}$.

In the model, additional variables are needed to distinguish between IRES generation within the range that can be guaranteed with a reasonable certainty, and IRES generation on top of the level that can be guaranteed with reasonable certainty. The original equation (Eq. (35) ) is now replaced by following set of equations and inequalities:

$$
\begin{aligned}
& \text { gen }_{\text {gri }, p, t}+\text { curt }_{\text {gri }, v, p, i, t}=\operatorname{cap}_{\text {gri }}^{\text {new }} C F_{\text {gri }, v, p, i, t} \quad \forall g r i \in \mathcal{G R \mathcal { I }}, p \in \mathcal{P}, t \in \mathcal{T} \text {, } \\
& \text { gen }_{\text {gri }, p, t}=\text { gen }_{\text {gri } i, v, p, i, t}^{\text {certain }}+\text { gen }_{\text {gri } i, v, p, i, t}^{\text {uncertain }} \quad \forall \text { gri } \in \mathcal{G R}, p \in \mathcal{P}, t \in \mathcal{T}, \\
& \operatorname{gen}_{\text {gri } i, v, p, i, t}^{\text {certain }} \leq \operatorname{cap}_{\text {gri }}^{\text {new }} C F_{\text {gri }, v, p, i, t}\left(1-\alpha_{\text {gri }}\right) \quad \forall g r i \in \mathcal{G} \mathcal{R} \mathcal{I}, p \in \mathcal{P}, t \in \mathcal{T}, \\
& \text { gen }_{\text {gri }, v, p, i, t}^{\text {uncertain }} \leq \operatorname{cap}_{\text {gri }}^{\text {new }} C F_{\text {gri }, v, p, i, t} \alpha_{\text {gri }} \quad \forall \text { gri } \in \mathcal{G} \mathcal{R} \mathcal{I}, p \in \mathcal{P}, t \in \mathcal{T},
\end{aligned}
$$

In line with Fig. 4 the required amount of operating reserves to deal with IRES forecast errors corresponds to gen $n_{g r i, v, p, i, t}^{u n c e r t a i n}$. This is adapted for in Eq. (16)-117).

The provision of upward reserves by IRES is restricted by the difference between the generation level that can be guaranteed with a reasonable certainty and the scheduled generation (see Fig. 5):

$$
\sum_{r \in \mathcal{R}} r_{r, g r i, p, t}^{+} \leq \operatorname{cap}_{\text {gri }}^{\text {new }} C F_{g r i, v, p, i, t}\left(1-\alpha_{\text {gri }}\right)-\operatorname{gen}_{\text {gri }, v, p, i, t}^{\text {certain }} \quad \forall g r i \in \mathcal{G} \mathcal{R} \mathcal{I}, p \in \mathcal{P}, t \in \mathcal{T}
$$

Additional constraints are needed to avoid that reserves are provided whenever the total generation of an intermittent generation source is effectively below the level that can be guaranteed with a reasonable certainty. Up to now, the model allows reducing $g_{\text {en }}^{\text {certain }}, i, p, t$ at the expense of increasing gen $_{g r i, v, p, i, t}^{\text {uncertain }}$. As such, upward reserves could be provided to any type of upward reserve. This comes at the expense of increasing the demand for operating reserves to cope with forecast errors (since gen uncertain is increased). 
However, depending on the required activation time used, these variable renewable forecast error reserves (VRFER) might be less expensive than other types of reserves. For this reason, additional constraints are incorporated, which only allow the provision of upward reserves by IRES whenever the total IRES generation level is below the level that can be guaranteed with a reasonable accuracy (or thus, whenever gen gri,ertain $=0$ ). To this end, an additional binary variable $z_{g r i, v, p, i, t}$ is introduced which indicates whether the upward reserves can be provided by IRES:

$$
\begin{gathered}
r_{r, g r i, p, t}^{+} \leq M z_{\text {gri } i, p, i, t} \quad \forall r \in \mathcal{R}, \text { gri } \in \mathcal{G} \mathcal{R} \mathcal{I}, p \in \mathcal{P}, t \in \mathcal{T}, \\
\text { gen }_{\text {gri }, v, p, i, t}^{\text {uncertain }} z_{\text {gri }, v, p, i, t}=0 \quad \forall \text { gri } \in \mathcal{G R} \mathcal{I}, p \in \mathcal{P}, t \in \mathcal{T}
\end{gathered}
$$

, where $M$ represents a very big number. However, the latter constraint is non-linear. Two additional constraints and an additional binary variable $y_{g r i, v, p, i, t}$ are introduced to replace the latter constraint and avoid the non-linearity:

$$
z_{g r i, v, p, i, t} \leq y_{g r i, v, p, i, t} \quad \forall g r i \in \mathcal{G} \mathcal{R} \mathcal{I}, p \in \mathcal{P}, t \in \mathcal{T},
$$

$$
\operatorname{gen}_{\text {gri }, v, p, i, t}^{\text {uncrtain }} \leq\left(1-y_{\text {gri }, v, p, i, t}\right) \operatorname{cap}_{\text {gri }}^{\text {new }} C F_{\text {gri }, v, p, i, t} \alpha_{\text {gri }} \quad \forall g r i \in \mathcal{G R} \mathcal{I}, p \in \mathcal{P}, t \in \mathcal{T} .
$$

Whenever $y_{g r i, v, p, i, t}$ is equal to one, Eq. 79 forces $g e n_{g r i, v, p, i, t}^{u n c e r t a i n}$ to 0 . In this case, $z_{g r i, v, p, i, t}$ can be set to 1 , and upward reserves can be provided. In contrast, whenever gen $n_{g r i, v, p, i, t}$ does not equal 0, Eq. 79 forces $y_{g r i, v, p, i, t}$ to be 0 , which according to Eq. (78) ensures that $z_{g r i, v, p, i, t}$ equals zero. Finally, via Eq. (76), this implies that no upward reserves can be provided.

\section{Case study description}

All simulations in this paper are performed for a system loosely inspired by the German electricity system for the year 2050. The optimization model is used in a greenfield mode, meaning that the focus is on a single year and no existing capacity is considered. Two different scenarios are considered to consider the relationship between the composition of the thermal generation fleet, on the one hand, and the impact of not considering technical constraints, on the other hand. In the 'Nuc' scenario, relatively inflexible nuclear plants are part of the capacity mix whereas in the 'No nuc' scenario, no nuclear plants are in the mix. To get these two different capacity mixes, a different tax on GHG emissions is imposed. In the 'No nuc' scenario, a GHG tax of $30 € /$ ton is used, whereas in the 'Nuc' scenario, a high tax of $100 € /$ ton is used. Additionally, both scenarios employ a support for renewables of $50 € / \mathrm{MWh}$.

In this paper, we limit ourselves to two scenarios, both having a high share of IRES but differing in the flexibility of the thermal generation fleet. This because the literature indicated that neglecting UC constraints has the highest impact for scenarios with high shares of IRES and is dependent on the capacity mix considered 26, 39, 12. We acknowledge that the results presented in this paper are to some degree dependent on the 
choice of scenarios and that additional scenarios could be considered (e.g., considering existing capacity, degree of coal power plants in the mix, etc.). However, it must be noted that the aim of this paper is to explore the sensitivities related to certain assumptions regarding the availability of flexibility and the need for flexibility in future power systems, rather than analyzing the relationship between the system composition and the impact of neglecting UC constraints. For a more detailed analysis of the relationship between the system composition and the impact of UC constraints, we refer to [26, 39, 12.

The capacity factor time series for onshore and offshore wind generation and solar photovoltaic (PV) generation are taken from the EMHIRES data sets [40] for Germany, as provided by the Strategic Energy Technologies Information System (SETIS) of the European Commission. These time series are scaled according to the endogenously determined capacity of wind and PV. The electricity demand time series for Germany are taken from the transparency platform provided by the European Network of Transmission System Operators for Electricity (ENTSO-E) [41]. To manage the computational complexity in the reference case, the year is represented by eight representative weeks, which are selected using our model developed in [10. These representative weeks are modeled at an hourly resolution 8

Data regarding investment costs, fixed and variable operations and maintenance costs, as well as life times and lead times are taken from [43, 30. Additionally, fuel prices are taken from the new policies scenario from the International Energy Agency's World Energy Outlook 2015 [44. An exception is made for the fuel related costs for nuclear plants, which are adopted from 30. A discount rate of $5 \%$ is used to annualize the investment costs. All data is presented in Tables 9.11.

\begin{tabular}{lccccccc}
\hline Technology & $\begin{array}{c}\text { Invest- } \\
\text { ment } \\
\text { cost } \\
{\left[\frac{k E U R}{k W_{e}}\right]}\end{array}$ & $\begin{array}{c}\text { FOM } \\
{\left[\frac{E U R}{\left(k W_{e} a\right)}\right]}\end{array}$ & $\begin{array}{c}\text { VOM } \\
{\left[\frac{E U R}{M W h_{e}}\right]}\end{array}$ & $\begin{array}{c}\text { Life } \\
\text { time } \\
{[a]}\end{array}$ & $\begin{array}{c}\text { Lead } \\
\text { time } \\
{[a]}\end{array}$ & $\begin{array}{c}\text { Effi- } \\
\text { ciency } \\
{[\%]}\end{array}$ & $\begin{array}{c}\text { Availa- } \\
\text { bility } \\
{[\%]}\end{array}$ \\
\hline NUC & 5.00 & 42 & 5 & 50 & 7 & 36 & 85 \\
COAL SC & 1.70 & 33 & 6 & 35 & 4 & 49 & 85 \\
COAL SC CCS & 2.02 & 34 & 20 & 35 & 5 & 40 & 85 \\
CCGT & 0.86 & 20 & 4 & 25 & 2 & 64 & 85 \\
CCGT CCS & 1.09 & 39 & 10 & 25 & 3 & 53 & 85 \\
OCGT & 0.57 & 17 & 4 & 15 & 2 & 45 & 85 \\
Onsh. Wind & 1.11 & 21 & - & 25 & 1 & - & - \\
Offsh. Wind & 2.10 & 60 & - & 25 & 1 & - & - \\
Sol. PV & 0.78 & 12 & - & 30 & 1 & - & - \\
\hline
\end{tabular}

Table 9: Economic characteristics of the considered thermal and renewable technologies. The VOM costs of the CCS technologies include capture and transportation costs. Data is taken from [43, 30].

\footnotetext{
${ }^{8}$ The impact of sub-hourly modeling has been examined in 42 , where it is shown that the impact of modeling sub-hourly dynamics is relatively small.
} 


\begin{tabular}{lcc}
\hline Characteristic & PHS & BAT \\
\hline Investment cost power $\left[\frac{k E U R}{k W_{e}}\right]$ & 1.15 & - \\
Investment cost energy $\left[\frac{k E U R}{M W h}\right]$ & 98.2 & 337.4 \\
FOM $\left[\frac{E U R}{\left(k W_{e} a\right)}\right]$ & 3.4 & 16.9 \\
VOM $\left[\frac{E U R}{M W h_{e}}\right]$ & 5 & - \\
Life time $[a]$ & 80 & 10 \\
Lead time $[a]$ & 4 & 2 \\
Round-trip Efficiency [\%] & 75 & 90 \\
Availability [\%] & 97 & 97 \\
Minimum discharge duration $[h]$ & 4 & 1 \\
Maximum discharge duration $[h]$ & 16 & 1 \\
\hline
\end{tabular}

Table 10: Economic characteristics of the considered storage technologies. The reported efficiencies correspond to the round-trip efficiency. Data is taken from [43, 30, 34].

\begin{tabular}{llll}
\hline Fuel & Coal & Natural gas & Uranium \\
\hline Price $\left[E U R / M W h_{p}\right]$ & 23.3 & 32.2 & 3 \\
\hline
\end{tabular}

Table 11: Fuel prices.

\section{Results and discussion}

First, Section 5.1 focuses on the impact of neglecting technical constraints for varying assumptions regarding the available flexibility. Subsequently, Section 5.2 specifically analyzes the impact of the reserve requirements. Finally, Section 5.3 explores the sensitivity of investments in storage technologies.

\subsection{Cycling capabilities and other flexibility providers}

Fig. 6a and Fig. 6b respectively display the projected total annual system costs and the capacity mix for all scenarios, flexibility cases and for all three models (MIP, LP and $\mathrm{MO}$ ). In addition, the generation mix and $\mathrm{CO}_{2}$ emissions are presented in Tab. 12. The impact of neglecting technical constraints can be seen by comparing the solution of the MIP model to that of the MO model.

By looking at the different cases for the available flexibility in Fig. 6, it can clearly be observed that the impact of neglecting technical constraints is highly sensitive to the assumptions taken regarding the flexibility of thermal generators and the availability of other sources of flexibility.

Most notably, whenever the model has the option to invest in alternative sources of flexibility (storage in this case), the integration of detailed technical constraints systematically has only a minor impact on both the projections of the total system cost and the capacity mix (small differences between the solution of the MIP and the MO model in the Stor Inflex and the Stor Flex cases). Depending on the scenario and the assumed flexibility of thermal generators, the projected system costs are underestimated by a mere $2.5-5 \%$ if investments in PHS and batteries are considered. Moreover, investments 


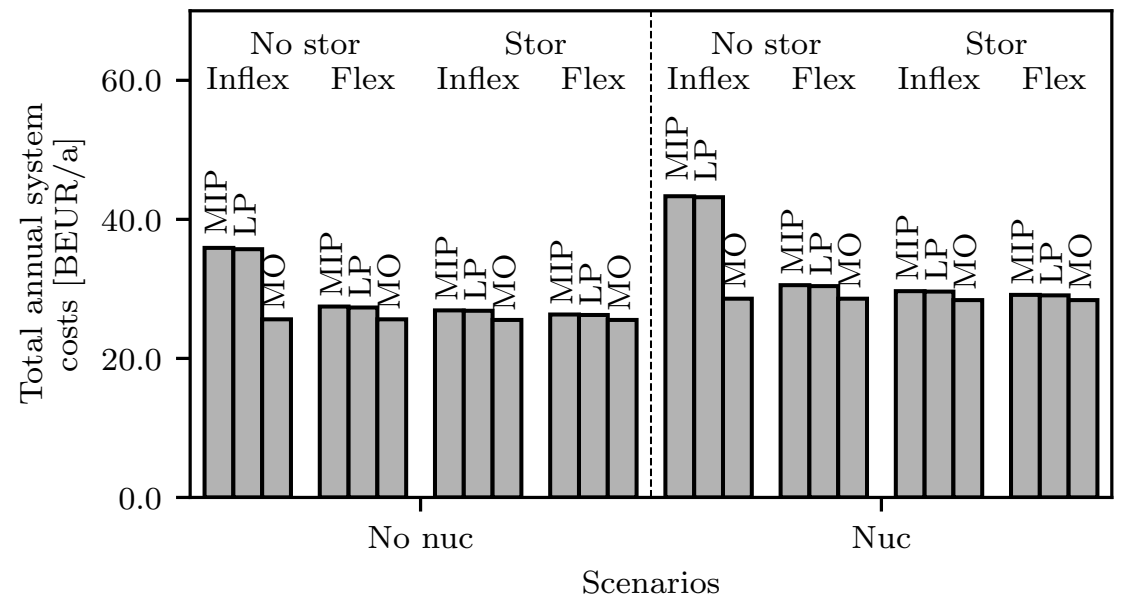

(a)
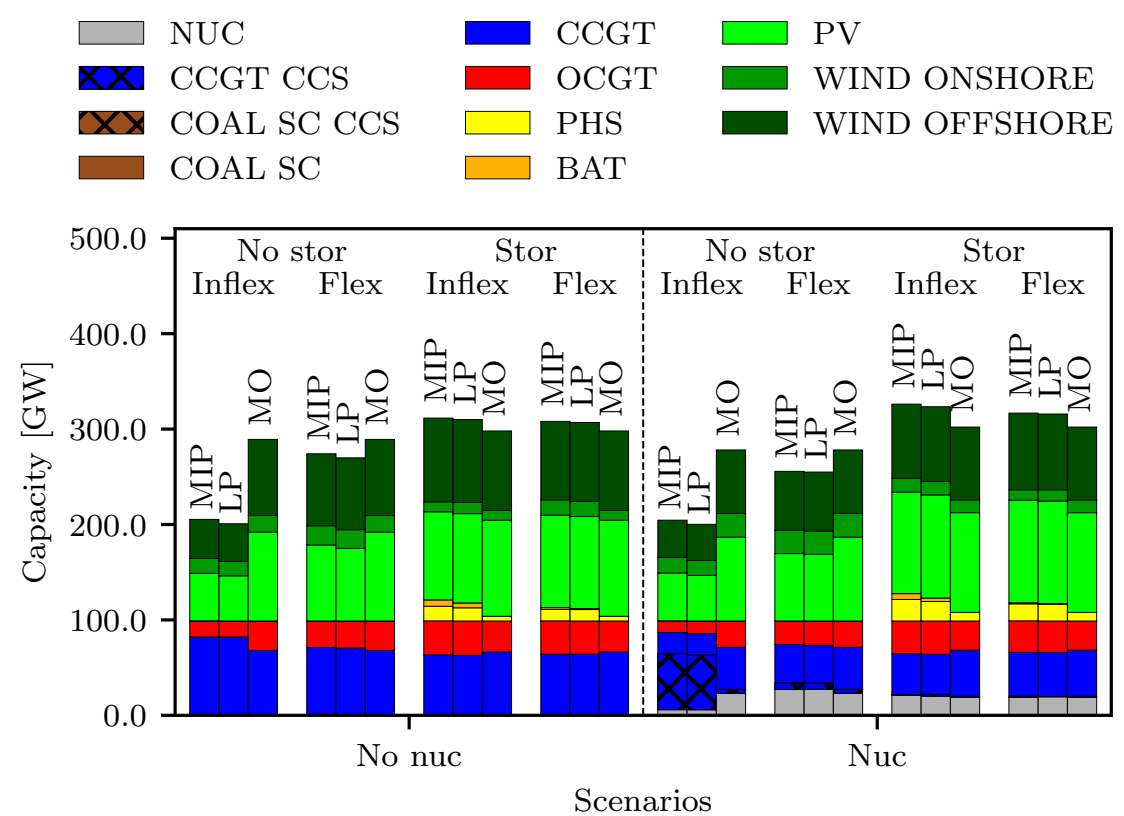

(b)

Figure 6: The projected total system cost (a) and the capacity mix (b) for the different scenarios (horizontal axis) and sensitivity cases regarding the consideration of alternative flexibility providers and the cycling capabilities of thermal generators. An overview of these sensitivity cases is presented in Tab. 4 The impact of integrating unit commitment constraints in generation expansion planning models can be assessed by comparing the solution of the 'MIP' model to that of the 'MO' model. 


\begin{tabular}{|c|c|c|c|c|c|c|c|c|c|c|c|c|}
\hline & \multicolumn{12}{|c|}{ No Nuc } \\
\hline & \multicolumn{3}{|c|}{$\begin{array}{l}\text { No stor } \\
\text { Inflex }\end{array}$} & \multicolumn{3}{|c|}{$\begin{array}{l}\text { No stor } \\
\text { Flex }\end{array}$} & \multicolumn{3}{|c|}{$\begin{array}{l}\text { Stor } \\
\text { Inflex }\end{array}$} & \multicolumn{3}{|c|}{$\begin{array}{l}\text { Stor } \\
\text { Flex }\end{array}$} \\
\hline & MIP & LP & MO & MIP & LP & MO & MIP & LP & MO & MIP & LP & MO \\
\hline NUC & 0 & 0 & 0 & 1.3 & 0 & 0 & 0 & 0 & 0 & 0 & 0 & 0 \\
\hline CCGT CCS & 0 & 0 & 0 & 0 & 0 & 0 & 0 & 0 & 0 & 0 & 0 & 0 \\
\hline CCGT & 62.7 & 63.5 & 32.9 & 34.7 & 36.3 & 32.9 & 29.5 & 29.5 & 31.2 & 30.2 & 30.2 & 31.2 \\
\hline OCGT & 0.4 & 0.4 & 0.5 & 1.4 & 1.5 & 0.5 & 0.4 & 0.4 & 0.4 & 0.5 & 0.5 & 0.4 \\
\hline PV & 7.2 & 7.1 & 13.0 & 12.6 & 12.7 & 13.0 & 13.6 & 14.5 & 14.5 & 14.6 & 15.3 & 14.5 \\
\hline WIND ON. & 4.0 & 4.9 & 4.0 & 4.2 & 4.3 & 4.0 & 2.8 & 3.8 & 2.5 & 4.4 & 4.7 & 2.5 \\
\hline $\begin{array}{l}\text { WIND OFF. } \\
\text { TOT RES }\end{array}$ & $\begin{array}{c}25.7 \\
(36.9)\end{array}$ & $\begin{array}{l}24.1 \\
(36.1)\end{array}$ & $\begin{array}{c}49.6 \\
(66.6)\end{array}$ & $\begin{array}{c}45.8 \\
(62.6)\end{array}$ & $\begin{array}{c}45.2 \\
(62.2)\end{array}$ & $\begin{array}{c}49.6 \\
66.6)\end{array}$ & $\begin{array}{l}53.7 \\
(70.1)\end{array}$ & $\begin{array}{c}51.8 \\
(70.1)\end{array}$ & $\begin{array}{c}51.4 \\
(68.4)\end{array}$ & $\begin{array}{c}50.3 \\
(69.3)\end{array}$ & $\begin{array}{c}49.3 \\
(69.3)\end{array}$ & $\begin{array}{c}51.4 \\
(68.4)\end{array}$ \\
\hline \multirow[t]{4}{*}{$\mathrm{CO}_{2} \mathrm{em}}$. & 122.3 & 123.5 & 58.5 & 66.3 & 69.2 & 58.5 & 55.4 & 55.5 & 55.7 & 56.0 & 55.9 & 55.7 \\
\hline & \multicolumn{12}{|c|}{ Nuc } \\
\hline & \multicolumn{3}{|c|}{$\begin{array}{l}\text { No stor } \\
\text { Inflex }\end{array}$} & \multicolumn{3}{|c|}{$\begin{array}{c}\text { No stor } \\
\text { Flex }\end{array}$} & \multicolumn{3}{|c|}{$\begin{array}{l}\text { Stor } \\
\text { Inflex }\end{array}$} & \multicolumn{3}{|c|}{$\begin{array}{l}\text { Stor } \\
\text { Flex }\end{array}$} \\
\hline & MIP & LP & MO & MIP & LP & MO & MIP & $\mathrm{LP}$ & $\mathrm{MO}$ & MIP & LP & MO \\
\hline NUC & 6.2 & 6.2 & 20.8 & 27.4 & 27.8 & 20.8 & 18.6 & 17.8 & 16.8 & 16.7 & 17.4 & 16.8 \\
\hline CCGT CCS & 51.6 & 51.7 & 2.9 & 4.6 & 4.2 & 2.9 & 0.4 & 1.2 & 0.6 & 0.8 & 0.0 & 0.6 \\
\hline CCGT & 6.1 & 6.7 & 13.5 & 11.3 & 11.3 & 13.5 & 12.1 & 11.8 & 15.1 & 13.2 & 13.6 & 15.1 \\
\hline OCGT & 0.2 & 0.2 & 0.3 & 0.7 & 0.7 & 0.3 & 0.2 & 0.2 & 0.2 & 0.2 & 0.2 & 0.2 \\
\hline PV & 7.2 & 6.8 & 13.2 & 10.8 & 11.1 & 13.2 & 16.6 & 16.7 & 16.1 & 16.5 & 16.9 & 16.1 \\
\hline WIND ON. & 4.2 & 4.6 & 7.2 & 6.5 & 6.4 & 7.2 & 4.5 & 4.5 & 3.9 & 3.3 & 3.9 & 3.9 \\
\hline $\begin{array}{l}\text { WIND OFF. } \\
\text { TOT RES }\end{array}$ & $\begin{array}{c}24.5 \\
(35.9)\end{array}$ & $\begin{array}{c}23.8 \\
(35.2)\end{array}$ & $\begin{array}{c}42.1 \\
(62.5)\end{array}$ & $\begin{array}{c}38.7 \\
(56.0)\end{array}$ & $\begin{array}{c}38.5 \\
(56.0)\end{array}$ & $\begin{array}{c}42.1 \\
(62.5)\end{array}$ & $\begin{array}{c}47.6 \\
(68.7)\end{array}$ & $\begin{array}{c}47.8 \\
(69.0)\end{array}$ & $\begin{array}{c}47.3 \\
(67.3)\end{array}$ & $\begin{array}{c}49.3 \\
(69.1)\end{array}$ & $\begin{array}{c}48.0 \\
(68.8)\end{array}$ & $\begin{array}{c}47.3 \\
(67.3)\end{array}$ \\
\hline $\mathrm{CO}_{2} \mathrm{em}$. & 28.6 & 29.8 & 25.1 & 24.1 & 23.8 & 25.1 & 23.0 & 22.7 & 27.3 & 25.0 & 25.4 & 27.3 \\
\hline
\end{tabular}

Table 12: Generation shares and $\mathrm{CO}_{2}$ emissions in the No Nuc and the Nuc scenario. Generation shares of the different technologies are expressed as a percentage of the net generated electricity. $\mathrm{CO}_{2} \mathrm{emissions}$ are expressed in Mton $\mathrm{CO} 2$ equivalent per year

in both thermal generators and IRES are hardly impacted. These relatively small differences in the capacity mix in the MIP and the MO model translate directly into small differences in the generation mix and the $\mathrm{CO}_{2}$ emissions, as can be seen in Tab. 12 .

In contrast, if thermal generators are considered to be the only source of flexibility, the impact of neglecting the technical constraints can become significantly higher. Especially if thermal generators are also assumed to be relatively inflexible (No stor Inflex case), the impact of incorporating technical constraints on the projections of the total system costs becomes very high (29-34\% cost increase for the considered scenarios). In addition, far fewer investments in IRES and less flexible baseload technologies (nuclear in this case) can be observed if technical constraints are accounted for. In turn, this can lead to huge differences in the generation mix and the $\mathrm{CO}_{2}$ emissions, as shown in Tab. 12 . The question remains however whether the assumptions adopted in the No stor Inflex case are realistic.

Under the assumptions of having no alternative sources of flexibility, and limitedly flexible thermal generators, the value of flexibility can become extremely high. This becomes apparent from the high difference in the projected total annual system cost between the No stor Inflex case and the three other flexibility cases for both scenarios 
(see Fig. 6a). These high differences indicate that every source of flexibility, be it more flexible thermal generators, storage technology-types or other sources of flexibility, has the potential to reduce costs significantly (even if this source of flexibility would be highly expensive). For this reason, assuming that no source of flexibility will be found might be highly unrealistic.

To support this statement, Tab. 13 presents the annual total system costs in the No stor Inflex and the Stor Inflex case. Additionally, this table presents the annual total system costs in a simulation in which the investment cost of all storage technologytypes is doubled (indicated by Storx2 Inflex). This table shows that, even when storage technology-types would be twice as expensive, the total system costs would be significantly lower than those projected by a model in which storage technology-types would not be considered at all. Similarly, in such a flexibility-constrained system, it can be expected that thermal power plants would be designed to offer more flexibility [45, or simply operated more flexibly at the expense of higher wear and tear costs.

\begin{tabular}{cccc}
\hline Scenario & No stor Inflex & Stor Inflex & Storx2 Inflex \\
\hline No_nuc & 33.4 & 26.9 & 28.0 \\
Nuc & 43.3 & 29.7 & 31.2 \\
\hline
\end{tabular}

Table 13: Total annual system cost [BEUR/a] for different assumptions regarding the availability of storage technologies

We therefore recommend to account for other sources of flexibility and/or the ability to increase the flexibility of thermal power plants when incorporating detailed technical constraints in planning models. Integrating technical constraints with overly conservative assumptions regarding the available flexibility can introduce errors, both in terms of projecting the total system cost and deriving the optimal capacity mix, which can be significantly higher than the errors one would obtain if technical constraints would be completely omitted. A resulting pitfall when integrating technical constraints in planning models is that overly conservative assumptions are taken regarding the available flexibility and the need for flexibility. Under such assumptions, the challenge of integrating large shares of IRES can be strongly overestimated, which in turn can lead to a strong overestimation of the projection of the total system cost and a technology bias.

Another important observation which can be made based on the results from Fig. 66 is that both the assumptions made regarding the cycling capabilities of thermal generators and the availability of other flexibility providers have a significantly higher impact on the results than the impact of using continuous (LP model) rather than integer commitment variables (MIP model). In terms of projections of the total system cost, the maximum difference between the MIP and the LP model for the different scenarios and flexibility cases was found to be merely $0.52 \%$. However, the calculation time increases from an average (over the different scenarios and flexibility cases) of 2746 seconds in the MIP model to an average of less than 100 seconds in the LP model. A more detailed representation of the computational performance, including the impact on the number of variables and constraints is presented in Tab. 14 


\begin{tabular}{l|ccc|cc}
\hline Model variant & \# Eq. & \# Var. & $\begin{array}{c}\text { \# Discr. } \\
\text { var. }\end{array}$ & $\begin{array}{c}\text { Comp. } \\
\text { time }\end{array}$ & Speed-up \\
\hline MIP & 249,864 & 212,385 & 56,448 & 2524 & - \\
LP & 249,864 & 212,385 & 0 & 91 & 27.7 \\
MO & 22,869 & 26,910 & 0 & 1.5 & 1635 \\
\hline
\end{tabular}

(a) cases without storage

\begin{tabular}{l|ccc|cc}
\hline Model variant & \# Eq. & \# Var. & $\begin{array}{c}\text { \# Discr. } \\
\text { var. }\end{array}$ & $\begin{array}{c}\text { Comp. } \\
\text { time }\end{array}$ & Speed-up \\
\hline MIP & 357,412 & 295,741 & 75,264 & 2968 & - \\
LP & 356,068 & 295,741 & 0 & 102.6 & 28.9 \\
MO & 49,793 & 43,066 & 0 & 3.9 & 770.2 \\
\hline
\end{tabular}

(b) cases with storage

Table 14: Overview of the problem size and the computational performance of the model variants with different levels of technical detail in the cases without storage (a) and with storage (b). The number of equations, variables and discrete variables as well as the average computation time and speed-up are presented. The number of equations and variables presented correspond to the 'Nuc' scenario. In the 'No nuc' scenario, these values are slightly lower as the nuclear technology is excluded in this scenario. Computation times are expressed in seconds and speed-ups are expressed relative to the reference (MIP) model.

Given the magnitude of the sensitivity of the results to certain input data assumptions, the small impact of relaxing the integrality conditions of the commitment variables on the obtained results, and the high impact of relaxing the integrality conditions on the computation time, we argue that for expansion planning of large, interconnected power systems, integrating detailed technical constraints using integer commitment variables is in most cases not worthwhile. In this regard, given the limited impact of incorporating technical constraints, one could even argue to not include these constraints at all (or opt for a more simplified and less computationally demanding formulation of the technical constraints than in the LP model). Neglecting all technical constraints (MO model) further reduces the computation time significantly, to an average of less than 3 seconds.

In addition to addressing the question whether it is worthwhile to integrate (MIP) constraints in GEP models, a key takeaway of this analysis is that to address flexibility in GEP models, it is not sufficient to simply integrate UC constraints. The impact of certain data-related assumptions taken when integrating UC constraints (e.g., how flexible are future power plants, which types of flexibility providers are assumed to be present, how is their flexibility simulated and to what extent are these flexibility sources assumed to be capable of contributing to operating reserve requirements) is at least of an equal order of magnitude than the level of detail of representing the UC constraints.

A single exception for which integrating technical constraints has a high impact relates to investments in storage technology-types, and especially batteries. From Fig. 6b, it can be observed that if technical constraints are omitted, no or few investments in batteries and PHS can be observed, whereas significant investments in storage technology-types 
can be observed if technical constraints are incorporated.

An interesting side effect, although limited in magnitude, is that the models with integrated technical constraints counterintuitively tend to invest in slightly more renewables if storage technologies are included, as can be seen from the total generation share from renewable technologies in Tab. 12. This result follows from the fact that the main driver for the investments in storage technologies in a model with integrated UC constraints is to contribute to reserve provision. However, once these storage technologies are in place, they are also used to avoid start-ups, part-load efficiency losses and curtailment of renewable electricity. As such, the presence of these storage technologies, which are driven by the UC constraints, facilitates the integration of higher shares of RES.

\subsection{Characterization of operating reserve requirements}

This section analyzes the sensitivity to the assumptions made regarding the characterization of operating reserves when detailed technical constraints are considered. Tab. 15 shows the relative decrease in the projected total system cost for the considered operating reserve cases (with respect to the REF case), and this for all scenarios and flexibility cases.

\begin{tabular}{llcccc}
\hline Scenario & reserve case & $\begin{array}{c}\text { No stor } \\
\text { Inflex }\end{array}$ & $\begin{array}{c}\text { No stor } \\
\text { Flex }\end{array}$ & Stor Inflex & Stor Flex \\
\hline \multirow{3}{*}{ No nuc } & Curt & 17.5 & 2.0 & 0.0 & 0.0 \\
& REF-20\% & 5.5 & 0.8 & 0.3 & 0.2 \\
& No_op_res & 24.4 & 4.2 & 1.3 & 1.0 \\
\cline { 2 - 6 } Nuc & Curt & 21.3 & 0.6 & 0.0 & 0.0 \\
& REF-20\% & 7.0 & 0.6 & 0.3 & 0.3 \\
& No_op_res & 29.0 & 2.8 & 1.0 & 0.7 \\
\hline
\end{tabular}

Table 15: Relative decrease of the total system cost with respect to the REF case [\%]

From this table, it can be observed that integrating operating reserve requirements in GEP models can have a very high impact on the cost projections (No_op_res case). However, the impact of considering operating reserve requirements is strongly dependent on the assumptions taken regarding the available flexibility. If thermal generators are assumed to be rather flexible and particularly if storage-technology types can provide reserves, the impact of reserves on the projections of the total system costs becomes almost negligible. This highlights the importance of not only considering different sources of flexibility, but also their ability to contribute to meeting operating reserve requirements.

A second observation is that properly accounting for how curtailment impacts operating reserve requirements and the ability of IRES to provide upward reserves (Curt case) can significantly decrease the expected total system costs if other sources of flexibility are not considered. Again, this effect is most outspoken if thermal generators are assumed to be limitedly flexible. 
If there are high shares of IRES and thermal generators are assumed to be the only flexibility providers, ensuring sufficient operating reserves can become very expensive. This is due to multiple reasons. First, an increasing penetration of IRES increases the need for reserves to deal with possible deviations from the forecasted conditions. Second, with an increasing instantaneous generation of IRES, the number of thermal generators which need to be online to generate electricity is reduced. Therefore, a higher volume of reserves needs to be provided by fewer units. At moments of high IRES generation, a minimum number of spinning units needs to remain online in order to provide the reserve requirements. Since these units are bound to generate above a minimum threshold, i.e., the minimum operating point, curtailment of IRES generation will be required even though the entire demand is not served by IRES. At higher penetration levels of IRES, this need to curtail will occur more frequently. If thermal generators are assumed to be more flexible, ensuring sufficient operating reserves becomes less costly. In this regard, particularly the minimum operating point and the ramping capabilities play a key role. As the ramping capabilities increase and the minimum operating point decreases, thermal units can provide more reserves per unit of electricity generated. As a result, in periods of high wind and/or solar generation, IRES can provide a much higher fraction of the demand which strongly reduces the fuel costs.

Storage systems, such as pumped-hydro storages and batteries have the inherent advantage that they can provide upward reserves without having to be generating electricity. Batteries are sufficiently fast to provide reserves without having to be charging or discharging. Whereas this is not necessarily the case for PHS systems, PHS systems can still provide upward reserves while charging (i.e., pumping) by reducing the charging power? Particularly in systems with a high penetration of IRES, this offers the advantage that curtailment of IRES and the need to generate electricity using thermal generators can (to some extent) be avoided. Similarly, some other flexibility providers, such as active demand response (ADR), have similar properties. Specifically, during moments of high renewable infeed, it can be expected that price-responsive loads will be increased, thereby also increasing the options available to provide upward reserves by reducing certain loads in case of a rapid decrease of IRES electricity generation. It are these types of flexibility providers which have not been considered in the current literature assessing the impact of considering UC constraints in GEP models, and which are key for providing flexibility at a reasonable cost during periods of high IRES generation.

\subsection{Investments in storage technologies}

Fig. 7 shows the investments in storage technologies for the different scenarios, flexibility cases and operating reserves cases.

A first observation that can be made by comparing the investments in storage technologies in the REF case to those in the No_op_res case is that, despite the limited impact of operating reserve requirements on the total system cost when storage systems can provide reserves, these operating reserve requirements form a determining factor for

\footnotetext{
${ }^{9}$ It must be noted that some of the existing pumped-hydro storages are fixed-speed machines which can only provide upward reserves in generation mode. In contrast, more modern adjustable speed machines can provide reserves both in pumping and turbining modes 34 .
} 


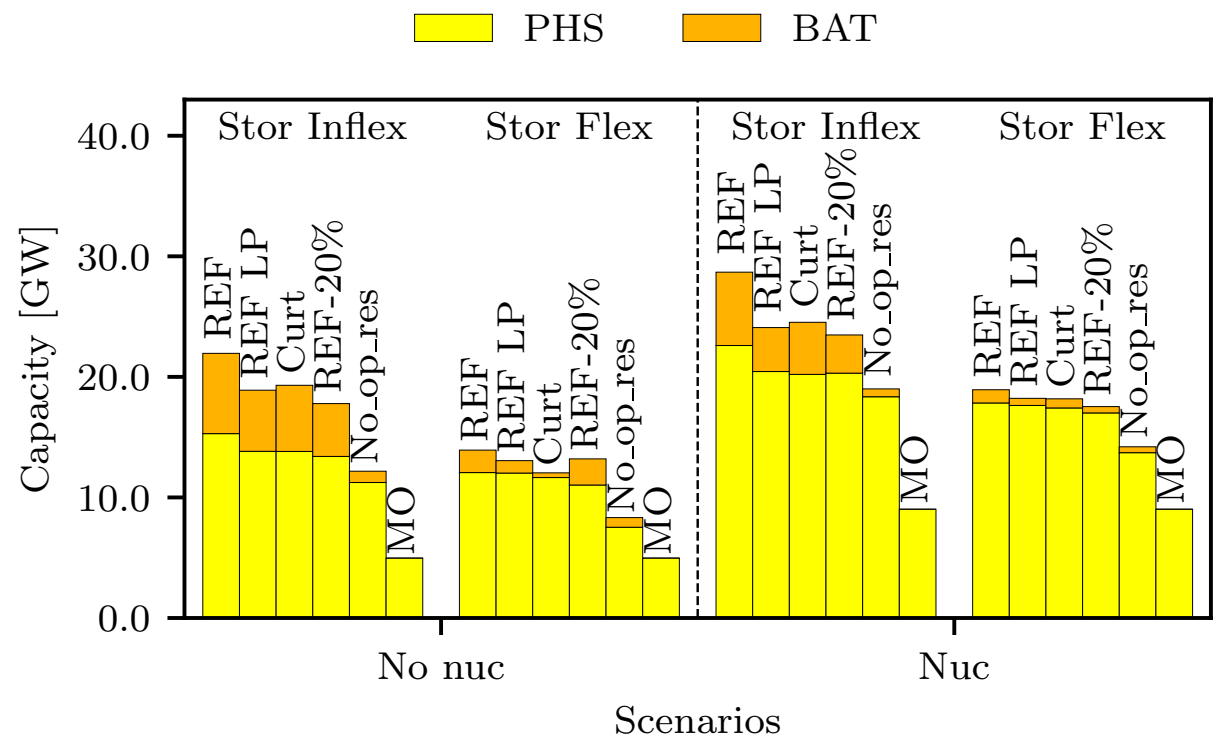

Figure 7: Investments in pumped hydro storage (PHS) and batteries (BAT) for the different scenarios and sensitivity cases. The scenarios are presented on the horizontal axis. The sensitivity cases with respect the cycling capabilities of thermal generators are presented at the top of the figure. These sensitivity cases are summarized in Tab. 4 The sensitivity cases with respect to the characterization of operating reserve requirements are shown above each bar. These sensitivity cases are summarized in Tab. 8

investments in storage technologies in general and batteries in particular. These findings are in line with van Stiphout, Brijs, Belmans and Deconinck [46].

A second observation that can be made is that the investments in storage technologies (and batteries in particular) are highly sensitive to the assumptions taken regarding the flexibility of thermal generators (Stor Inflex versus Stor Flex case). To a lesser degree, investments in storage technologies are also sensitive to the assumptions taken for characterizing operating reserve requirements ( $R E F$ versus Curt and $R E F-20 \%$ case). The impact of the assumptions regarding the cycling capabilities of thermal power plants and the sizing of operating reserve requirements are shown to be respectively of an order of magnitude higher, and a similar order of magnitude than the impact of using continuous instead of integer commitment variables.

\section{Summary and conclusions}

In the context of an increasing penetration of intermittent renewable energy sources, concerns regarding flexibility have stimulated modelers to integrate detailed unit commitment constraints in generation expansion planning models. The recent literature has also assessed the impact of these unit commitment constraints in generation expansion 
planning models. Under the assumptions taken, it has been concluded that these constraints have a significant impact on the results, and should therefore not be neglected. However, in this literature, some assumptions have been made of which the impact has not been explored. First, in the current literature assessing the impact of unit commitment constraints, thermal generators are often considered to be the only source of flexibility. Second, the sensitivity of the results to the assumed cycling capabilities of thermal generators and the characterization of operating reserve requirements has not been investigated.

This paper contributes to the existing literature by revisiting the impact of unit commitment constraints in generation expansion planning models for a variety of assumptions regarding the availability of other flexibility providers, the cycling capabilities of thermal generators and the characterization of operating reserve requirements.

A first important conclusion is that unit commitment constraints in generation expansion planning models have a limited impact on both the projections of the total system cost, and the investments in thermal generators and renewable capacity if other technologies than thermal generators are considered to provide flexibility. In contrast, integrating unit commitment constraints (in combination with operating reserve requirements) is shown to have a significant impact on the investments in storage technologies (which can be generalized to investments in other dedicated flexibility providers). We therefore conclude that if the generation expansion planning model is not used to specifically investigate the role of storage or other dedicated flexibility providers, integrating unit commitment constraints is not critical for the obtained results. In contrast, if the generation expansion planning model is used to investigate the role of such dedicated flexibility providers, considering these unit commitment constraints is vital.

A second important conclusion of this paper is that, based on the current literature, one might overestimate the impact of integrating unit commitment constraints in generation expansion planning models due to the fact that alternative sources of flexibility have not been considered. As such, the presented analysis also exposes a potential pitfall for generation expansion planning models integrating unit commitment constraints. Namely, if overly conservative assumptions are taken regarding the flexibility of thermal generators and no other flexibility providers are being considered (or they cannot contribute to the provision of operating reserves), the integration of unit commitment constraints can lead to a strong overestimation of the challenges related to integrating large shares of intermittent renewable energy sources. This can in turn lead to a high overestimation of the projected system costs and a strong technology bias. It was shown that these errors can be significantly higher than the errors introduced by not integrating unit commitment constraints. We therefore recommend considering different sources of flexibility and/or the ability to increase the flexibility of thermal generators when integrating unit commitment constraints in planning models.

A third conclusion is that the investments in these dedicated flexibility providers (pumped-hydro storage and batteries in this paper) are highly sensitive to the assumptions taken regarding the cycling capabilities of thermal generators and the characterization of the operating reserve requirements. We therefore recommend researchers in- 
vestigating the optimal level of storage or other flexibility providers in the system to be aware of this sensitivity.

A final important finding of this paper relates to the formulation used to integrate unit commitment constraints. Specifically, it is shown that the errors introduced by using a formulation which integrates all unit commitment constraints but uses continuous instead of integer commitment variables, are significantly lower than the impact of the choice of cycling capabilities of thermal generators, and of about an equal order of magnitude as the impact of the assumptions typically adopted to characterize reserve requirements. We therefore conclude that, unless one is specifically interested in the role of dedicated flexibility providers ánd the sensitivities to the availability of other sources of flexibility, the cycling capabilities of thermal generators and the characterization of reserve requirements are actively explored, there is little value in integrating full unit commitment constraints with integer commitment variables.

As a final note, we remark that a lot of attention in the literature has gone to tractable integration of unit commitment constraints. In the light of our findings, namely the importance of representing alternative flexibility providers and the sensitivity to operating reserve requirements, we argue that integrating unit commitment constraints in planning models in itself is not sufficient to achieve good and robust investment decisions. A first modeling challenge to be addressed is to integrate accurate models of alternative flexibility providers in long-term planning models. A second modeling challenge relates to the characterization of operating reserve requirements, including both the sizing and the characterization (e.g., full activation time), in future power systems. A third and related challenge is to develop methods to endogenously approximate these reserve requirements in planning models. A fourth and final challenge relates to dealing with the inherent uncertainty regarding the future need for flexibility and the availability of certain flexibility providers (e.g., residential demand response, flexibility provided by electric vehicles).

\section{Acknowledgements}

The research of Kris Poncelet has been supported by a $\mathrm{PhD}$ grant provided by VITO.

\section{References}

[1] A. Pina, C. A. Silva, P. Ferrão, High-resolution modeling framework for planning electricity systems with high penetration of renewables, Applied Energy 112 (2013) 215-223. doi:10.1016/j .apenergy. 2013.05 .074

[2] N. E. Koltsaklis, A. S. Dagoumas, State-of-the-art generation expansion planning: A review, Applied Energy 230 (2018) 563 - 589. doi:https://doi.org/10.1016/j.apenergy.2018.08.087. URL http://www.sciencedirect.com/science/article/pii/S0306261918312583

[3] D. S. Kirschen, J. Ma, V. Silva, R. Belhomme, Optimizing the flexibility of a portfolio of generating plants to deal with wind generation, in: 2011 IEEE PES General Meeting, 2011, pp. 1-7. doi: 10.1109/PES.2011.6039157

[4] J. Ma, V. Silva, R. Belhomme, D. S. Kirschen, L. F. Ochoa, Evaluating and planning flexibility in sustainable power systems, IEEE Transactions on Sustainable Energy 4 (1) (2013) 200-209. doi:10.1109/TSTE. 2012.2212471

[5] F. J. de Sisternes, Investment model for renewable electricity systems (IMRES): an electricity generation capacity expansion formulation with unit commitment constraints, MIT Center for Energy and Environmental Policy Research Working Paper 2013-06 (2013). 
[6] N. E. Koltsaklis, M. C. Georgiadis, A multi-period, multi-regional generation expansion planning model incorporating unit commitment constraints Applied Energy 158 (2015) 310 - 331. doi: https://doi.org/10.1016/j.apenergy.2015.08.054

URL http://www.sciencedirect.com/science/article/pii/S0306261915009873

[7] S. Pereira, P. Ferreira, A. Vaz, Generation expansion planning with high share of renewables of variable output Applied Energy 190 (2017) 1275 - 1288. doi:https://doi.org/10.1016/j.apenergy. 2017.01 .025

URL http://www.sciencedirect.com/science/article/pii/S0306261917300338

[8] F. J. de Sisternes, M. D. Webster, Optimal Selection of Sample Weeks for Approximating the Net Load in Generation Planning Problems (Jan. 2013)

URL http://citeseerx.ist.psu.edu/viewdoc/download?doi=10.1.1.362.8358\&rep=rep1\&type= pdf

[9] P. Nahmmacher, E. Schmid, L. Hirth, B. Knopf, Carpe Diem: A Novel Approach to Select Representative Days for Long-Term Power System Models with High Shares of Renewable Energy Sourcesidoi: 10.2139/ssrn.2537072

URL http://dx.doi.org/10.2139/ssrn.2537072

[10] K. Poncelet, H. Höschle, E. Delarue, A. Virag, W. Dhaeseleer, Selecting representative days for capturing the implications of integrating intermittent renewables in generation expansion planning problems, IEEE Transactions on Power Systems 32 (3) (2017) 1936-1948. doi:10.1109/TPWRS. 2016.2596803

[11] A. Flores-Quiroz, R. Palma-Behnke, G. Zakeri, R. Moreno, A column generation approach for solving generation expansion planning problems with high renewable energy penetration Electric Power Systems Research 136 (2016) 232 - 241. doi:https://doi.org/10.1016/j.epsr.2016.02. 011 URL http://www.sciencedirect.com/science/article/pii/S0378779616300177

[12] B. S. Palmintier, M. D. Webster, Impact of operational flexibility on electricity generation planning with renewable and carbon targets, IEEE Transactions on Sustainable Energy 7 (2) (2016) 672-684. doi:10.1109/TSTE.2015.2498640

[13] B. S. Palmintier, M. D. Webster, Heterogeneous unit clustering for efficient operational flexibility modeling, IEEE Transactions on Power Systems 29 (3) (2014) 1089-1098. doi:10.1109/TPWRS. 2013.2293127

[14] B. Palmintier, Flexibility in generation planning: Identifying key operating constraints, in: Power Systems Computation Conference (PSCC), 2014, IEEE, 2014, pp. 1-7.

[15] L. Zhang, T. Capuder, P. Mancarella, Unified unit commitment formulation and fast multi-service lp model for flexibility evaluation in sustainable power systems, in: 2016 IEEE Power and Energy Society General Meeting (PESGM), 2016, pp. 1-1. doi:10.1109/PESGM.2016.7741184

[16] B. Hua, R. Baldick, J. Wang, Representing operational flexibility in generation expansion planning through convex relaxation of unit commitment, IEEE Transactions on Power Systems 33 (2) (2018) 2272-2281. doi:10.1109/TPWRS.2017.2735026

[17] M. Welsch, P. Deane, M. Howells, B. Ó. Gallachóir, F. Rogan, M. Bazilian, H.-H. Rogner, Incorporating flexibility requirements into long-term energy system models a case study on high levels of renewable electricity penetration in ireland, Applied Energy 135 (0) (2014) $600-615$. doi:http://dx.doi.org/10.1016/j.apenergy.2014.08.072

URL http://www.sciencedirect.com/science/article/pii/S0306261914008836

[18] C. D. Jonghe, E. Delarue, R. Belmans, W. Dhaeseleer, Determining optimal electricity technology mix with high level of wind power penetration, Applied Energy 88 (6) (2011) 2231 - 2238. doi: http://dx.doi.org/10.1016/j.apenergy. 2010.12.046

URL http://www.sciencedirect.com/science/article/pii/S0306261910005660

[19] C. Batlle, P. Rodilla, An enhanced screening curves method for considering thermal cycling operation costs in generation expansion planning, IEEE Transactions on Power Systems 28 (4) (2013) 3683-3691. doi:10.1109/TPWRS.2013.2249540

[20] I. Staffell, R. Green, Is there still merit in the merit order stack? the impact of dynamic constraints on optimal plant mix, IEEE Transactions on Power Systems 31 (1) (2016) 43-53. doi:10.1109/ TPWRS.2015.2407613

[21] S. Collins, J. P. Deane, K. Poncelet, E. Panos, R. C. Pietzcker, E. Delarue, B. Ó Gallachóir, Integrating short term variations of the power system into integrated energy system models: A methodological review, Renewable and Sustainable Energy Reviews 76 (Supplement C) (2017) 839 -856. doi:https://doi.org/10.1016/j.rser.2017.03.090 URL http://www.sciencedirect.com/science/article/pii/S1364032117304264 
[22] K. Poncelet, Long-term energy-system optimization models: Capturing the challenges of integrating intermittent renewable energy sources and assessing the suitability for descriptive scenario analyses Ph.D. thesis, KU Leuven (2018).

URL https://lirias.kuleuven.be/bitstream/123456789/604991/1/thesis_final.pdf

[23] J. Deane, A. Chiodi, M. Gargiulo, B. P. O. Gallachóir, Soft-linking of a power systems model to an energy systems model Energy 42 (1) (2012) 303 - 312, 8th World Energy System Conference, WESC 2010. doi:http://dx.doi.org/10.1016/j.energy.2012.03.052. URL http://www.sciencedirect.com/science/article/pii/S0360544212002551

[24] K. Poncelet, E. Delarue, D. Six, J. Duerinck, W. D'haeseleer, Impact of the level of temporal and operational detail in energy-system planning models, Applied Energy 162 (2016) 631-643. doi:10.1016/j.apenergy.2015.10.100

[25] A. Belderbos, E. Delarue, Accounting for flexibility in power system planning with renewables International Journal of Electrical Power \& Energy Systems 71 (0) (2015) 33 - 41. doi:http: //dx.doi.org/10.1016/j.ijepes.2015.02.033.

URL http://www.sciencedirect.com/science/article/pii/S0142061515001167

[26] A. Shortt, J. Kiviluoma, M. O’Malley, Accommodating variability in generation planning, IEEE Transactions on Power Systems 28 (1) (2013) 158-169. doi:10.1109/TPWRS.2012.2202925

[27] B. Palmintier, M. Webster, Impact of unit commitment constraints on generation expansion planning with renewables, in: 2011 IEEE Power and Energy Society General Meeting, 2011, pp. 1-7. doi:10.1109/PES.2011.6038963

[28] I. F. Abdin, E. Zio, An integrated framework for operational flexibility assessment in multi-period power system planning with renewable energy production, Applied Energy 222 (2018) 898 - 914. doi:https://doi.org/10.1016/j.apenergy.2018.04.009

URL http://www.sciencedirect.com/science/article/pii/S0306261918305518

[29] A. Nogales, S. Wogrin, E. Centeno, Impact of technical operational details on generation expansion in oligopolistic power markets, IET Generation, Transmission Distribution 10 (9) (2016) 2118-2126. doi:10.1049/iet-gtd.2015.1148

[30] A. Schröder, F. Kunz, J. Meiss, R. Mendelevitch, C. von Hischhausen, Data documentation- current and prospective costs of electricity generation until 2050, dIW Working Paper, https://www.diw. de/documents/publikationen/73/diw_01.c.424566.de/diw_datadoc_2013-068.pdf

[31] K. V. den Bergh, E. Delarue, Cycling of conventional power plants: Technical limits and actual costs Energy Conversion and Management 97 (Supplement C) (2015) 70 - 77. doi:https://doi. org/10.1016/j.enconman.2015.03.026

URL http://www.sciencedirect.com/science/article/pii/S0196890415002368

[32] N. Kumar, P. Besuner, S. Lefton, A. D., D. Hilleman, Power plant cycling costs Tech. rep., NREL (2012).

URL https://www.nrel.gov/docs/fy12osti/55433.pdf

[33] J. Meus, K. Poncelet, E. Delarue, Applicability of a clustered unit commitment model in power system modeling, IEEE Transactions on Power Systems PP (99) (2017) 1-1. doi:10.1109/TPWRS. 2017.2736441

[34] MWH, Technical Analysis of Pumped Storage and Integration with Wind Power in the Pacific Northwest - Final Report, Tech. rep., MWH (2009).

[35] T. Mai, C. Barrows, A. Lopez, E. Hale, M. Dyson, K. Eurek, Implications of Model Structure and Detail for Utility Planning: Scenario Case Studies Using the Resource Planning Model Tech. rep., National Renewable Energy Laboratory (NREL), nREL/TP-6A20-63972 (2015). URL https://www.nrel.gov/docs/fy15osti/63972.pdf

[36] W. Short, P. Sullivan, T. Mai, M. Mowers, C. Uriarte, N. Blair, D. Heimiller, A. Martinez, Regional Energy Deployment System (ReEDS). Tech. rep., National Renewable Energy Laboratory (NREL), nREL/TP-6A20-46534 (2011). URL http://www.nrel.gov/docs/fy12osti/46534.pdf

[37] R. Loulou, U. Remne, A. Kanudia, A. Lehtila, G. Goldstein, Documentation for the TIMES model: Part I, ETSAP (April 2005).

[38] K. Poncelet, E. Delarue, D. Six, W. D'haeseleer, Myopic optimization models for simulation of investment decisions in the electric power sector, in: 13th International Conference on the European Energy Market (EEM), 2016, pp. 1-9. doi:10.1109/EEM.2016.7521261

[39] B. S. Palmintier, Incorporating operational flexibility into electric generation planning - impacts and methods for system design and policy analysis Ph.D. thesis, Massachusetts Institute of Technology (2013).

URL http://hdl.handle.net/1721.1/79147 
[40] Strategic Energy Technologies Information System, European Commission, EMHIRES data sets, available at https://setis.ec.europa.eu/EMHIRES-datasets (2017).

[41] European Network of Transmission System Operators for Electricity (ENTSO-E), ENTSO-E Transparency Platform (2017).

URL https://transparency.entsoe.eu/

[42] J. Deane, G. Drayton, B. O. Gallachóir, The impact of sub-hourly modelling in power systems with significant levels of renewable generation, Applied Energy 113 (2014) 152 - 158. doi: https: //doi.org/10.1016/j.apenergy.2013.07.027

URL http://www.sciencedirect.com/science/article/pii/S030626191300593X

[43] S. Simoes et al., The JRC-EU-TIMES model - Assessing the long-term role of the SET Plan Energy technologies, Tech. rep., JRC's Institute for Energy and Transport (2013).

[44] International Energy Agency (IEA), World Energy Outlook 2015

URL https://www . iea.org/publications/freepublications/publication/WE02015.pdf

[45] H. Holttinen, A. Tuohy, M. Milligan, E. Lannoye, V. Silva, S. Müller, L. Sö", The flexibility workout: Managing variable resources and assessing the need for power system modification, IEEE Power and Energy Magazine 11 (6) (2013) 53-62. doi:10.1109/MPE.2013.2278000

[46] A. van Stiphout, T. Brijs, R. Belmans, G. Deconinck, Quantifying the importance of power system operation constraints in power system planning models: A case study for electricity storage Journal of Energy Storage 13 (2017) 344 - 358. doi:https://doi.org/10.1016/j.est.2017.07.003 URL http://www.sciencedirect.com/science/article/pii/S2352152X16302201 